\title{
OPEN An innovative green synthesis approach of chitosan nanoparticles and their inhibitory activity against phytopathogenic Botrytis cinerea on strawberry leaves
}

\author{
Noura El-Ahmady El-Naggar ${ }^{1 \bowtie}$, WesamEldin I. A. Saber $\mathbb{B}^{2}$, Amal M. Zweil ${ }^{3}$ \& \\ Shimaa I. Bashir ${ }^{4}$
}

Green synthesis is a newly emerging field of nanobiotechnology that offers economic and environmental advantages over traditional chemical and physical protocols. Nontoxic, eco-friendly, and biosafe materials are used to implement sustainable processes. The current work proposes a new biological-based strategy for the biosynthesis of chitosan nanoparticles (CNPs) using Pelargonium graveolens leaves extract. The bioconversion process of CNPs was maximized using the response surface methodology. The best combination of the tested parameters that maximized the biosynthesis process was the incubation of plant extract with $1.08 \%$ chitosan at $50.38{ }^{\circ} \mathrm{C}$ for $57.53 \mathrm{~min}$., yielding $9.82 \pm 3 \mathrm{mg}$ CNPs/mL. Investigation of CNPs by SEM, TEM, EDXS, zeta potential, FTIR, XRD, TGA, and DSC proved the bioconversion process's success. Furthermore, the antifungal activity of the biosynthesized CNPs was screened against a severe isolate of the phytopathogenic Botrytis cinerea. CNPs exerted efficient activity against the fungal growth. On strawberry leaves, $25 \mathrm{mg} \mathrm{CNPs} / \mathrm{mL}$ reduced the symptoms of gray mold severity down to $3 \%$. The higher concentration of CNPs $(50 \mathrm{mg} / \mathrm{mL}$ ) was found to have a reverse effect on the infected area compared with those of lower concentrations ( 12.5 and $25 \mathrm{mg}$ CNPs $/ \mathrm{mL}$ ). Therefore, additional work is encouraged to reduce the harmful side effects of elevated CNPs concentrations.

Recent advances in the synthesis of diverse nano-molecules of varied sizes, shapes, and functionalities have established nanotechnology as an essential technology in a variety of disciplines ${ }^{1}$. Thus, nanotechnology has been revolutionizing almost all realms in the life sciences and many high-technology industries ${ }^{2}$. Nanoparticles, in contrast to bulk materials, have a greater specific exterior area and a high reaction activity. Thus, have been demonstrated to impact many living cells, including microbial ones ${ }^{1}$.

The natural chitosan polymer (polyglucosamine (1-4)-2-amino- $\beta$-D glucose) originates from the whole or partial deacetylation of chitin and occurs as a linear biopolymer of $\mathrm{D}$-glucosamine and $\mathrm{N}$-acetyl glucosamine units $^{2}$. Chitosan is biodegradable, soluble in acidic conditions, and carries a positive charge due to the free amino groups on its polymeric chains ${ }^{3,4}$. A growing interest is being focused on the reformation and application of chitosan, of which, a broad spectrum of antimicrobial properties was reported against several pathogens, such as Phytophthora infestans.

Chitosan has long been the preferred material to produce nanoparticles in a variety of applications. Compared to other sources, natural chitosan-based nanoparticles (CNPs) have several beneficial features (replenishability, biocompatibility, biodegradability, nontoxicity, high permeability toward biological membranes, and environmental safety), and are thus preferred for a variety of applications, e.g., antimicrobial activities ${ }^{2,3,6}$.

${ }^{1}$ Department of Bioprocess Development, Genetic Engineering and Biotechnology Research Institute, City of Scientific Research and Technological Applications (SRTA-City), New Borg El-Arab City 21934, Alexandria, Egypt. ${ }^{2}$ Microbial Activity Unit, Department of Microbiology, Soils, Water and Environment Research Institute, Agricultural Research Center, Giza 12619, Egypt. ${ }^{3}$ Plant Biotechnology Department, Genetic Engineering and Biotechnology Research Institute, University of Sadat City, Sadat City, Egypt. ${ }^{4}$ Department of Plant Protection and Biomolecular Diagnosis, Arid Land Cultivation Research Institute, City of Scientific Research and Technological Applications (SRTA-City), New Borg El-Arab City 21934, Alexandria, Egypt. ${ }^{\boxplus e m a i l}$ nouraalahmady@yahoo.com 

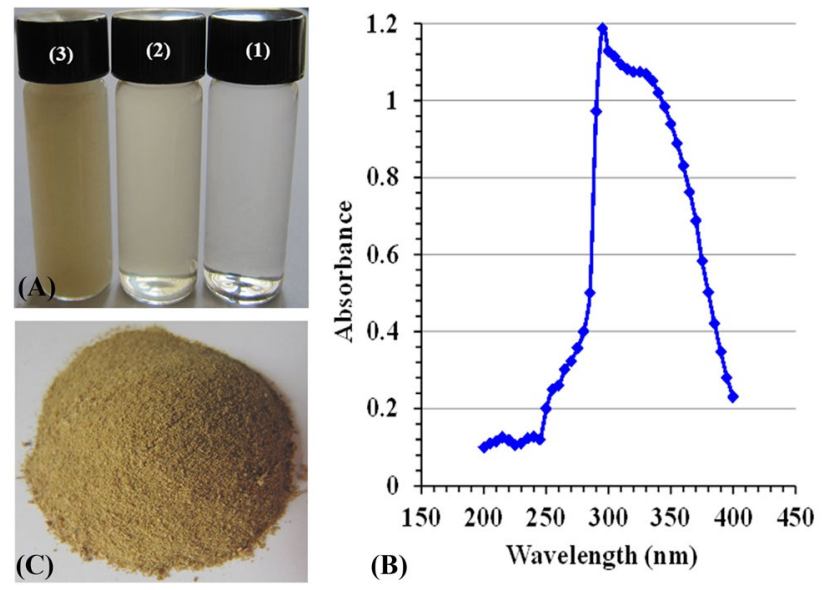

Figure 1. (A) Visible observation of chitosan nanoparticle biosynthesis (1, chitosan solution; 2, Pelargonium graveolens extract; 3, biosynthesized chitosan nanoparticles), (B) UV-Vis spectrum of chitosan nanoparticles, (C) dried chitosan nanoparticles biosynthesized using Pelargonium graveolens leaf extract.

Contrary to the physical and chemical procedures, green synthesis or phytofabrication of nano-molecules offers several advantages, including economization, fastness, safeness, cleanness, and environmentally acceptable, importantly, the reductive capabilities of the proteins and metabolites of the biological entity can easily convert a metal ion into nanoparticle form ${ }^{7-9}$.

Rose geranium or sweet-scented geranium (Pelargonium graveolens L'Her., family: Geraniaceae) has been successfully proven to be a nonhazardous and environmentally accepted candidate for the rapid and efficient generation of nanomaterials $s^{7,8,10}$. The plant is a perennial, hermaphrodite, evergreen, flowering plant with a shrub-like appearance that can grow as tall as $1.5 \mathrm{~m}$. The strongly rose-scented leaves (Supplementary Figure S1) are deeply incised, velvety, and soft to the touch because of the glandular hairs. The plant has a pale soft pink flower ${ }^{11-13}$. Plant leaf and its oil has several therapeutic applications for the healing of several internal and external diseases ${ }^{11,14}$.

Botrytis cinerea is one of the most common and destructive fungal pathogens, infecting more than 200 plant species in subtropical and temperate environments, many of which have very economic significance (e.g., strawberries, grapes, and solanaceous vegetables). Botrytis cinerea causes gray mold disease, which is obvious on the surface as gray fluffy mycelium. The symptoms are distributed across plant organs and tissues, including postharvest fruit rot, blossom blight, and leaf blight. Botrytis cinerea produces a broad range of low-molecularweight metabolites that induce an oxidative attack of the tissue, aggregation of free radicals, and hypersensitive cell death of plant cells during cuticle penetration and the development of primary lesions $s^{15,16}$. The pathogen is primarily regulated using chemical fungicides, considering an environmental issue and the possibility of developing fungicide resistance, as well ${ }^{15,17}$. Thus, finding an alternative safe controlling procedure, such as CNP, is of great importance.

However, to the best of the authors' information, no work has been performed on the bioconversion of organic compounds, such as chitosan, into nanoparticles using biological systems. Therefore, Pelargonium graveolens was used in the current study because it represents a safe base for the biological preparation of CNPs. The current trial is an alternative economic and eco-friendly procedure designed to cover the chasm of CNP preparation based on biological systems as a novel approach for the phytofabrication of CNPs. The characterization of the biofabricated nanoparticles was itemized, likewise, the antifungal features of the generated CNPs were explored on $B$. cinerea as a fungal model.

\section{Results}

Primary detection of the generated CNPs. This study focused on a novel fabrication of CNPs from chitosan solution using Pelargonium graveolens leaf extract. Fig. 1A shows three vials containing solutions of chitosan, leaf extract, and biosynthesized chitosan nanoparticles after the biofabrication process. The phytofabrication process of CNPs was monitored and analyzed through a UV/VIS'S spectra range of 200-400 nm (Fig. 1B). The optical properties of the CNP biopolymer materials displayed a single sharp peak, and the maximum absorbance wavelength of CNPs at $295 \mathrm{~nm}$ was noticed. The gained CNPs after biosynthesized and drying are depicted in Fig. 1C.

Modeling the phytofabrication of CNP biosynthesis. To model the bioprocessing conditions of CNP biosynthesis, a statistical modulating design built on response surface methodology was generated. A matrix of Box-Behnken design (BBD), having 17 experimental runs, was used to optimize the phytofabrication process using $P$. graveolens leaf extract. The design matrix, including the real and coded levels of the three variables as well as the experimental and expected values of CNPs and the errors (residuals), are introduced in Table 1. The 17 experimental runs of the phytofabrication process of CNPs showed various responses, ranging from 4.61 to 


\begin{tabular}{|c|c|c|c|c|c|c|c|c|c|c|}
\hline \multirow[b]{2}{*}{ Std } & \multirow[b]{2}{*}{ Run } & \multicolumn{2}{|c|}{$\begin{array}{l}\text { Incubation } \\
\text { period }(\mathrm{min})\end{array}$} & \multicolumn{2}{|c|}{ Temperature $\left({ }^{\circ} \mathrm{C}\right)$} & \multicolumn{2}{|c|}{ Chitosan (\%) } & \multicolumn{2}{|c|}{\begin{tabular}{|l|} 
Chitosan \\
nanoparticles \\
biosynthesis (mg/ \\
mL)
\end{tabular}} & \multirow[b]{2}{*}{ Residuals } \\
\hline & & Coded & Actual & Coded & Actual & Coded & Actual & Actual & Predicted & \\
\hline 8 & 1 & 1 & 90 & 0 & 50 & 1 & 1.5 & 6.62 & 6.6 & 0.02 \\
\hline 6 & 2 & 1 & 90 & 0 & 50 & -1 & 0.5 & 4.61 & 4.51 & 0.10 \\
\hline 2 & 3 & 1 & 90 & -1 & 40 & 0 & 1 & 5.14 & 5.23 & -0.09 \\
\hline 11 & 4 & 0 & 60 & -1 & 40 & 1 & 1.5 & 6.19 & 6.13 & 0.07 \\
\hline 13 & 5 & 0 & 60 & 0 & 50 & 0 & 1 & 9.58 & 9.66 & -0.08 \\
\hline 7 & 6 & -1 & 30 & 0 & 50 & 1 & 1.5 & 6.51 & 6.61 & -0.10 \\
\hline 3 & 7 & -1 & 30 & 1 & 60 & 0 & 1 & 6.23 & 6.14 & 0.09 \\
\hline 15 & 8 & 0 & 60 & 0 & 50 & 0 & 1 & 9.51 & 9.66 & -0.15 \\
\hline 16 & 9 & 0 & 60 & 0 & 50 & 0 & 1 & 9.72 & 9.66 & 0.07 \\
\hline 14 & 10 & 0 & 60 & 0 & 50 & 0 & 1 & 9.76 & 9.66 & 0.10 \\
\hline 4 & 11 & 1 & 90 & 1 & 60 & 0 & 1 & 7.27 & 7.3 & -0.03 \\
\hline 10 & 12 & 0 & 60 & 1 & 60 & -1 & 0.5 & 4.88 & 4.95 & -0.07 \\
\hline 12 & 13 & 0 & 60 & 1 & 60 & 1 & 1.5 & 7.42 & 7.41 & 0.01 \\
\hline 5 & 14 & -1 & 30 & 0 & 50 & -1 & 0.5 & 5.91 & 5.93 & -0.02 \\
\hline 1 & 15 & -1 & 30 & -1 & 40 & 0 & 1 & 7.85 & 7.82 & 0.03 \\
\hline 17 & 16 & 0 & 60 & 0 & 50 & 0 & 1 & 9.72 & 9.66 & 0.06 \\
\hline 9 & 17 & 0 & 60 & -1 & 40 & -1 & 0.5 & 5.82 & 5.83 & -0.01 \\
\hline Variable & Code & -1 & 0 & 1 & & & & & & \\
\hline Incubation period $(\mathrm{min})$ & A & 30 & 60 & 90 & & & & & & \\
\hline Temperature $\left({ }^{\circ} \mathrm{C}\right)$ & B & 40 & 50 & 60 & & & & & & \\
\hline Chitosan concentration (\%) & $\mathrm{C}$ & 0.5 & 1 & 1.5 & & & & & & \\
\hline
\end{tabular}

Table 1. Box-Behnken design representing chitosan nanoparticle biosynthesis by Pelargonium graveolens as influenced by incubation period $\left(\mathrm{X}_{1}\right)$, initial $\mathrm{pH}$ level $\left(\mathrm{X}_{2}\right)$, and chitosan concentration $\left(\mathrm{X}_{3}\right)$.

\begin{tabular}{|c|c|c|c|c|c|}
\hline Source & Sum of squares & Degrees of freedom & Mean square & $F$-value & $P$-value \\
\hline \multicolumn{6}{|l|}{ Lack-of-fit tests } \\
\hline Linear & 48.41 & 9 & 5.38 & 455.22 & $<0.0001^{*}$ \\
\hline $2 \mathrm{FI}$ & 43.22 & 6 & 7.20 & 609.63 & $<0.0001$ \\
\hline Quadratic & 0.0461 & 3 & 0.0154 & 1.30 & 0.3896 \\
\hline \multicolumn{6}{|c|}{ The sequential model sum of squares } \\
\hline Linear vs Mean & 4.91 & 3 & 1.64 & 0.44 & 0.7288 \\
\hline 2FI vis Linear & 5.19 & 3 & 1.73 & 0.40 & 0.7562 \\
\hline Quadratic vis $2 \mathrm{FI}$ & 43.18 & 3 & 14.39 & 1079.02 & $<0.0001^{*}$ \\
\hline Source & Standard deviation & $\mathbf{R}^{2}$ & Adjusted- $\mathbf{R}^{2}$ & Predicted- $\mathrm{R}^{2}$ & PRESS \\
\hline \multicolumn{6}{|c|}{ Model summary statistics } \\
\hline Linear & 1.93 & 0.092 & -0.1175 & -0.3632 & 72.75 \\
\hline $2 \mathrm{FI}$ & 2.08 & 0.1893 & -0.2972 & -0.9311 & 103.06 \\
\hline Quadratic & 0.12 & 0.9983 & 0.9960 & 0.9848 & 0.81 \\
\hline
\end{tabular}

Table 2. Summary of model fitness for Box-Behnken design results for chitosan nanoparticle biosynthesis using Pelargonium graveolens leaf extract as affected by chitosan concentration (\%), initial pH level, temperature $\left({ }^{\circ} \mathrm{C}\right)$, and incubation period $(\mathrm{min})$. ${ }^{*}$ Significant values, PRESS: sum of squares of prediction error, two factors interaction: 2FI.

$9.72 \mathrm{mg} / \mathrm{mL}$. the highest value is a center point. However, the actual experimental results of CNPs were found to be in line with the predicted values that were generated based on the equation model.

Model selection. To select the most fitted model, the lack of fit, the sum of squares, and certain model statistics tests were compared for the linear, two-factor interaction, and quadratic models (Table 2). The quadratic model was preferred based on the lower values of probability $(P)$ value, standard deviation, and the sum of squares of prediction error (PRESS) and higher values of determination coefficient $\left(\mathrm{R}^{2}\right)$, adjusted- $\mathrm{R}^{2}$, and predicted- $R^{2}$. The quadratic model was the most fitted and therefore was selected for the molding process. 


\begin{tabular}{|c|c|c|c|c|c|c|c|}
\hline \multicolumn{2}{|l|}{ Source of variance } & \multirow{2}{*}{\begin{tabular}{|l|}
$\begin{array}{l}\text { Coefficient } \\
\text { estimate }\end{array}$ \\
9.66 \\
\end{tabular}} & \multirow{2}{*}{\begin{tabular}{|l|} 
Sum of squares \\
53.28 \\
\end{tabular}} & \multirow{2}{*}{\begin{tabular}{|l|}
$\begin{array}{l}\text { Degrees of } \\
\text { freedom }\end{array}$ \\
9 \\
\end{tabular}} & \multirow{2}{*}{$\begin{array}{l}\text { Mean Square } \\
5.92 \\
\end{array}$} & \multirow{2}{*}{$\begin{array}{l}F \text {-value } \\
443.82\end{array}$} & \multirow{2}{*}{$\begin{array}{l}P \text {-value } \\
<0.0001 \\
\end{array}$} \\
\hline Model & Intercept & & & & & & \\
\hline \multirow{3}{*}{ Linear effect } & A & -0.36 & 1.02 & 1 & 1.02 & 76.66 & $<0.0001$ \\
\hline & B & 0.10 & 0.08 & 1 & 0.08 & 6.00 & 0.0442 \\
\hline & $\mathrm{C}$ & 0.69 & 3.81 & 1 & 3.81 & 285.56 & $<0.0001$ \\
\hline \multirow{3}{*}{ Interaction effect } & $\mathrm{AB}$ & 0.94 & 3.52 & 1 & 3.52 & 263.58 & $<0.0001$ \\
\hline & $\mathrm{AC}$ & 0.35 & 0.497 & 1 & 0.497 & 37.26 & 0.0005 \\
\hline & $\mathrm{BC}$ & 0.54 & 1.18 & 1 & 1.18 & 88.26 & $<0.0001$ \\
\hline \multirow{3}{*}{ Quadratic effect } & $\mathrm{A}^{2}$ & -1.60 & 10.77 & 1 & 10.77 & 807.75 & $<0.0001$ \\
\hline & $\mathrm{B}^{2}$ & -1.43 & 8.67 & 1 & 8.67 & 649.71 & $<0.0001$ \\
\hline & $\mathrm{C}^{2}$ & -2.14 & 19.37 & 1 & 19.37 & 1451.93 & $<0.0001$ \\
\hline \multirow{2}{*}{ Error effect } & Lack of fit & & 0.05 & 3 & 0.0154 & 1.30 & 0.3896 \\
\hline & Pure error & & 0.05 & 4 & 0.0118 & & \\
\hline \multicolumn{2}{|l|}{$\mathrm{R}^{2}$} & 0.9983 & \multicolumn{3}{|c|}{ Standard deviation } & 0.12 & \\
\hline \multicolumn{2}{|l|}{ Adjusted- $\mathrm{R}^{2}$} & 0.996 & \multicolumn{3}{|c|}{ Mean } & 7.22 & \\
\hline \multicolumn{2}{|l|}{ Predicted- $\mathrm{R}^{2}$} & 0.9848 & \multicolumn{3}{|c|}{ Coefficient of variation, $\%$} & 1.60 & \\
\hline \multicolumn{2}{|l|}{ Adequate Precision } & 58.08 & & & & & \\
\hline
\end{tabular}

Table 3. Analysis of variance for chitosan nanoparticle biosynthesis using Pelargonium graveolens leaf extract as affected by initial $\mathrm{pH}$ level, the incubation period ( $\mathrm{min})$, chitosan concentration (\%), and temperature $\left({ }^{\circ} \mathrm{C}\right)$.

* Significant values, $F$ : Fisher's function, $P$ : Level of significance.
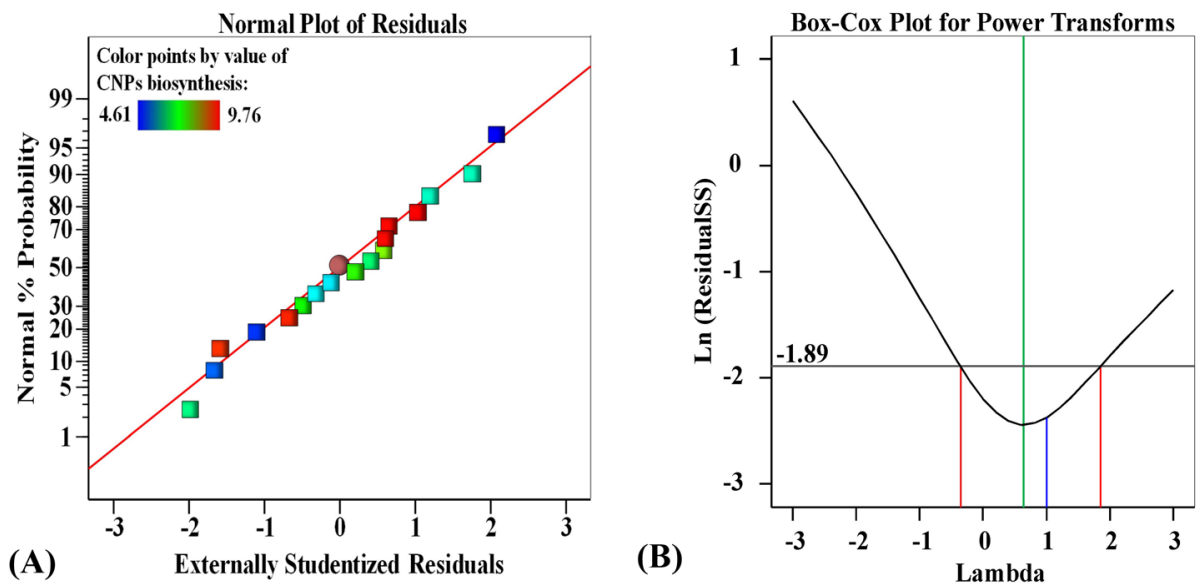

Color points by value of

CNPs biosynthesis:
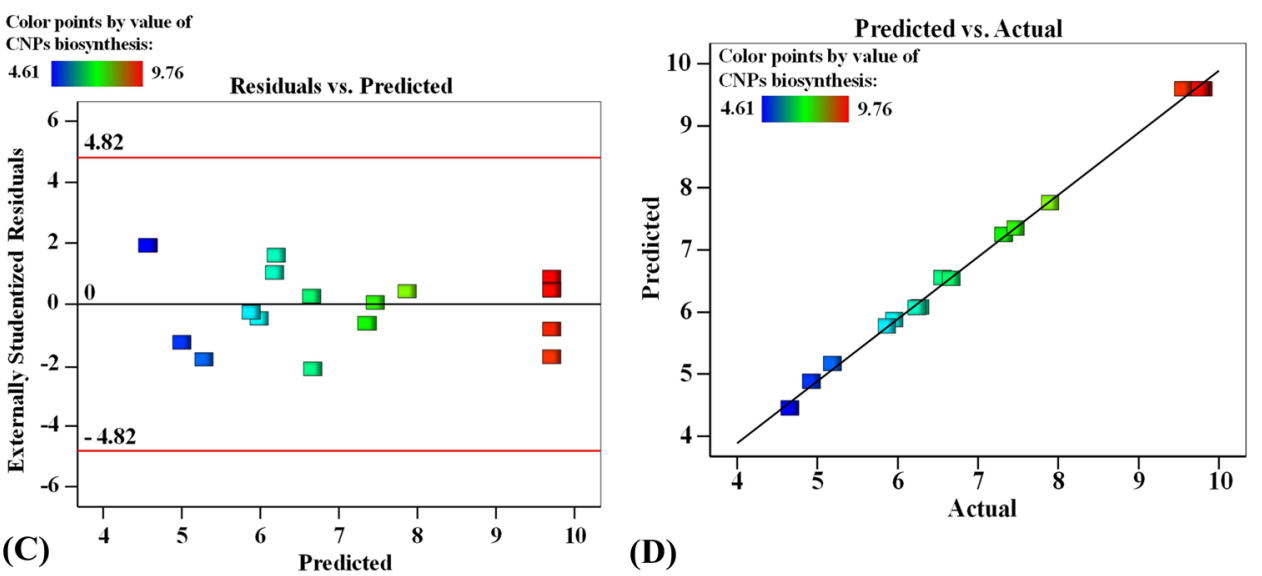

(D)

Figure 2. Normal probability plot of residuals (A), Box-Cox plot for power transformation (B), residuals versus predicted (C), and the predicted versus actual (D) values of chitosan nanoparticle biosynthesis using Pelargonium graveolens leaf extract. 

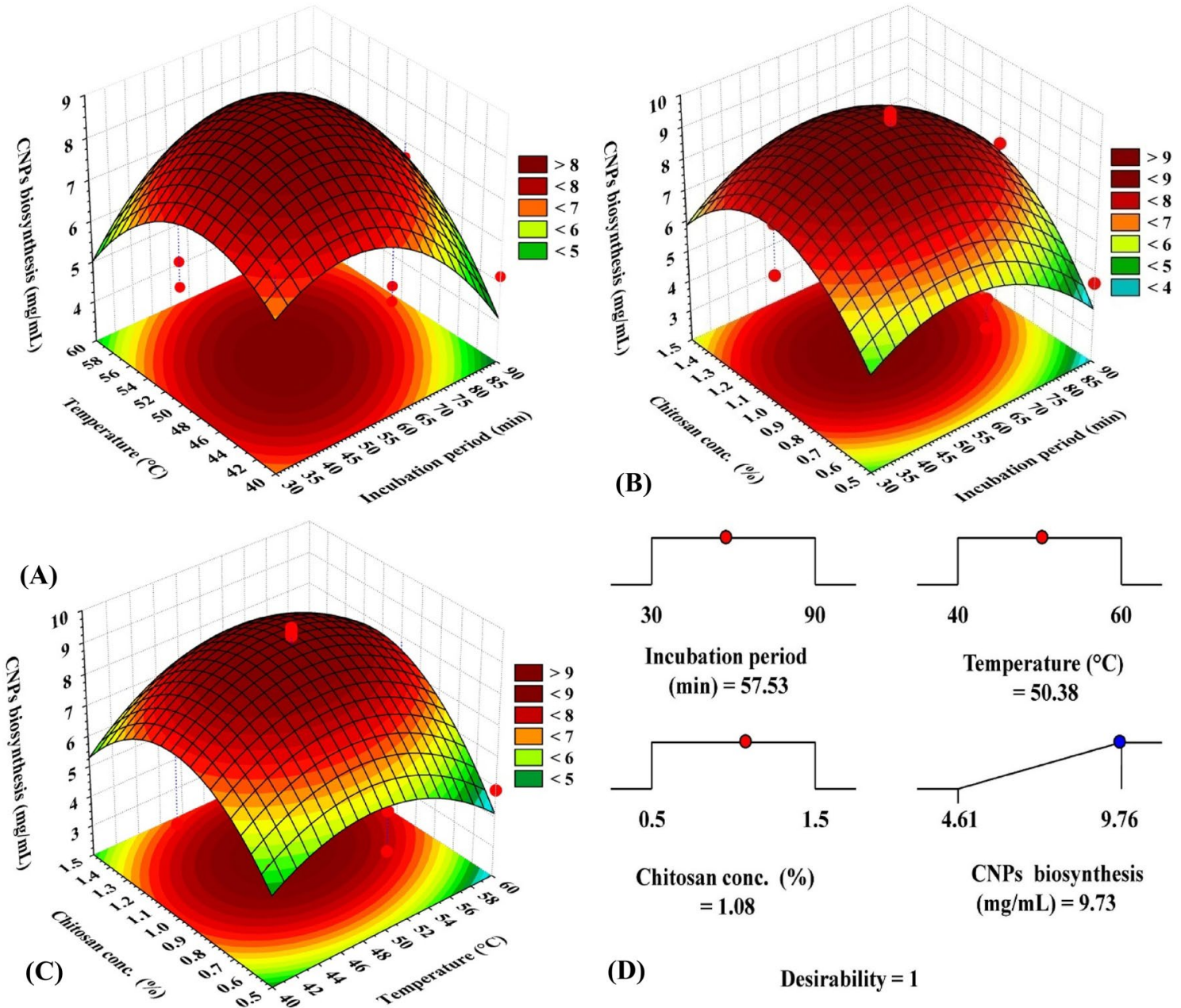

(D)

Desirability $=1$

Figure 3. Three-dimensional surface plot for chitosan nanoparticle biosynthesis by Pelargonium graveolens leaf extract, showing the interactive effects of each pair of factors, holding the third factor at the center point (A-C), and the optimization plot displaying the desirability function at the optimum predicted values (D) for the maximum chitosan nanoparticle biosynthesis using Pelargonium graveolens leaf extract.

Statistical and multiple regression analysis. To discover the significance of the BBD and its factors, both were exposed to the analysis of variance (ANOVA) and multiple regression analysis (Table 3 ). The overall model generated significant performance with a $P$-value $<0.05$. The linear, interaction, and quadratic effects followed the same significant trend. On the other hand, the lack-of-fit error recorded a higher $P$-value $>0.05$ that did not allow it to reach the significance threshold. Another, the values of the coefficient of variation (C.V. $=1.60)$ and adequate precision (58.08) are other evaluating parameters that showed the good performance of the model. All these are indicators of the significance of the model and its involved factors. The general mean and standard deviation values of the investigational runs are 94.95 and 1.53 , respectively.

Exploring ANOVA shows the aptness of the various individuals, interactions, and quadratic effects. The three types of $\mathrm{R}^{2}$ are adequate to suggest a high significance of both the model and its components. However, the linear, mutual interactions, or quadratic coefficient estimate showed various positive or negative significant responses, being negative for the incubation period and quadratic terms, whereas positive for the rest of the other terms (temperature and initial chitosan concentration) and all the interactions.

Model adequacy checking. The model adequacy was further checked to approve the appropriateness of the model. Mathematical statics were checked and described. The normal probability plot of the externally studentized residuals (Fig. 2A) displays that data points are concerted thoroughly along the straight line and follow the normal distribution without linearity. In addition, the depiction of the Box-Cox of the power transformation (Fig. $2 \mathrm{~B}$ ) shows that the current lambda $(\lambda)$ value is equal to one. However, the best $\lambda$ value (green line) was located between the confidence intervals (two red lines).

Likewise, the values of externally studentized residuals vis predicted values by the model were drawn. The plot (Fig. 2C) shows an equal scatter of the residual data above and below the 0 -axis. This pattern is ideal enough to prove the suitability of the BBD model. Similarly, the predicted values were drawn against the trial values to 


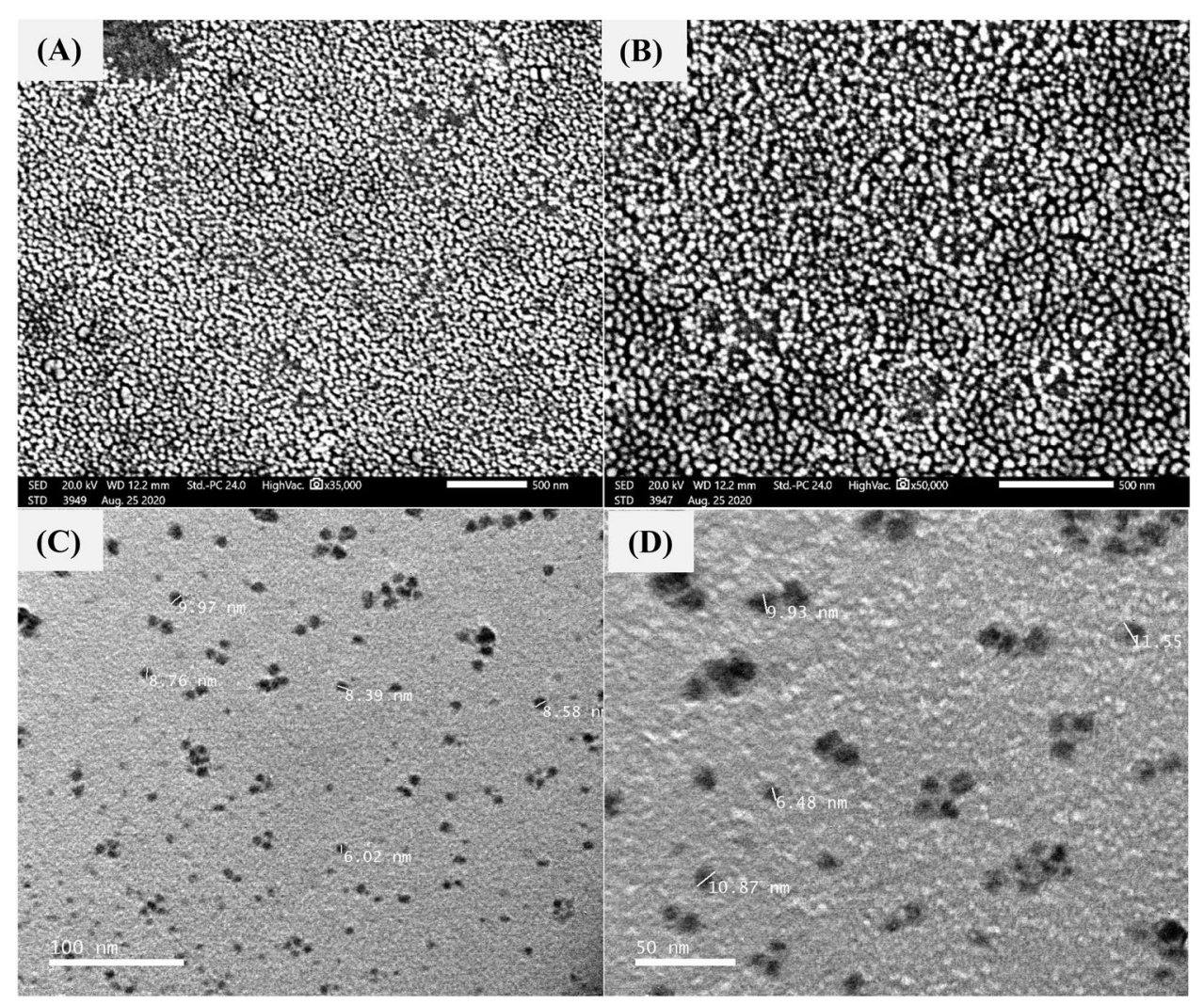

Figure 4. SEM (A,B) and TEM (C,D) micrographs of chitosan nanoparticles biosynthesized using Pelargonium graveolens leaf extract.

evaluate the aptness of the model (Fig. 2D). Again, the linear regression analysis displays better fitting of the model forecast points that lie nearer to the line of perfect prediction.

The three-dimensional (3D) surface plot. To explore the mutual influence of each pair of the tested parameters, 3D-surface plots of the simultaneous effect of the three independent factors on CNP biosynthesis using Pelargonium graveolens leaf extract were constructed. Fig. 3A-C illustrate two independent factors (at their central points) on the X-and Y-axes against the Z-axis (CNP biosynthesis). The maximum CNP biosynthesis was situated around the central points of temperature, incubation period, and chitosan concentration; out of these ranges, a marked decline in $\mathrm{CNP}$ biosynthesis was noticed.

Experimental validation of the model. The prime aim of the investigational strategy is to find the bestpredicted situations for obtaining the maximum CNPs. To find the best-predicted conditions for an extreme response, the desirability function was used, and the best combination of conditions was calculated based on the following prediction equation:

CNP biosynthesis $=9.66-0.36 \times$ incubation time $+0.10 \times$ temperature $+0.69 \times$ chitosan concentration +0.94 incubation time $\times$ temperature +0.35 incubation time $\times$ chitosan concentration +0.54 temperature $\times$ chitosan concentration -1.60 incubation time $^{2}-1.43$ temperature $^{2}-2.14$ chitosan concentration ${ }^{2}$.

According to the model's equation, this predicted value was achieved at the tested variables of $57.53 \mathrm{~min}$ (incubation period), $50.38^{\circ} \mathrm{C}$, and $1.08 \%$ (chitosan concentration). The highest theoretical (predicted) value of CNP biosynthesis was estimated to be $9.73 \mathrm{mg} / \mathrm{mL}$ (Fig. 3D). Such optimum points of the tested levels recorded a satisfactory desirability value (1.0).

To verify the green synthesis of CNPs by using P. graveolens leaf extract under the optimal predicted conditions, a triplicate set of experiments was carried out, and the experimental results were judged to the predicted values. The actual laboratory value of the green synthesis of CNPs by using $P$. graveolens leaf extract was $9.82 \pm 3 \mathrm{mg} / \mathrm{mL}$, verifying a high degree of model precision and confirming the validation of the model under the design matrix.

Electron microscopy investigation. The surface morphological structure was examined using scanning electron microscopy (SEM). The size, morphology, and structure were inspected at various magnification scales (Fig. 4A,B). The SEM image of the morphological construction of CNPs shows spherical-like particles, and their mild agglomerated state revealed a highly porous surface. Furthermore, the size of the nanoparticles also showed uniformity and homogeneity. 

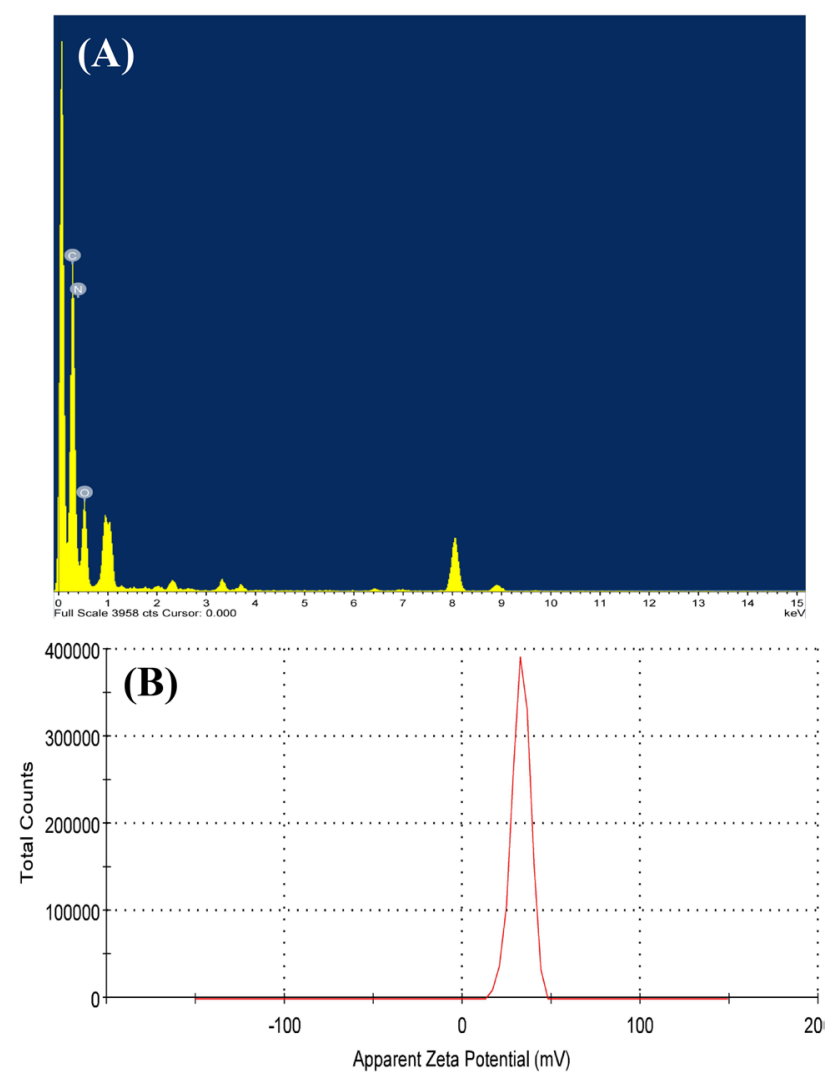

Figure 5. EDXS (A) and zeta potential (B) analyses of chitosan nanoparticles biosynthesized using Pelargonium graveolens leaf extract.

Another efficient tool for discovering the morphological structure of CNPs is transmission electron microscopy (TEM), which was performed at various magnification powers (Fig. 4C,D). TEM derives additional details, such as a particle's aggregation and agglomeration. The two-dimensional (2D) TEM image introduced a wider conception than SEM, especially regarding the non-aggregation and lower-agglomeration status of CNPs with a highly porous surface. Furthermore, the CNP size analysis showed a measurement from 6.02 to $10.87 \mathrm{~nm}$. It is of particular importance to note that both SEM and TEM were found to be complementary to the characterization of CNPs.

Energy-dispersive X-ray spectroscopy (EDXS) analysis. For further characterization, CNPs were explored for their elemental compositions using EDXS (Fig. 5A), which can quickly generate information about the kinds of elements, distributions, and concentrations. The EDXS spectra of CNPs confirmed the presence of the four elements that chitosan is composed of hydrogen, carbon, nitrogen, and oxygen. Such a composition represents the main elemental component of chitosan.

Zeta $(\zeta)$ potential analysis. The $\zeta$-potential analysis of the synthesized CNPs was investigated; nevertheless, $\zeta$-potential analysis is often the only available route for the description of double-layer properties. The depicted $\zeta$-potential (Fig. 5B) shows decent stability of the positively charged CNPs with a $\zeta$-potential of $+32.6 \pm 5.26 \mathrm{mV}$ at $25^{\circ} \mathrm{C}$. Furthermore, the $\zeta$-potential potential distribution has a single peak, indicating excellent uniformity of CNPs.

Fourier transform infrared (FTIR) investigation. CNPs were analyzed using FTIR (Fig. 6A) to explore the biomolecules for the possible occurrence of various functional groups that bind with CNPs due to reduction and stabilization. The detected intense bands were compared with standard values to classify the functional groups. The adsorbent spectra were measured in the range between 4500 and $500 \mathrm{~cm}^{-1}$. The characteristics of CNPs were shown by a broad absorption band. The FTIR spectra show absorption bands at 3736, 3442, 2350, $1572,1413,1072,914$, and $645 \mathrm{~cm}^{-1}$.

X-ray diffraction (XRD) analysis. The crystallographic structure of CNPs was investigated using XRD (Fig. 6B). After irradiating CNPs with incident X-rays, the intensities and scattering angles of the X-rays that left the CNPs were measured. XRD of CNPs showed three peaks at 2-theta of 13,19 , and $35^{\circ}$. 

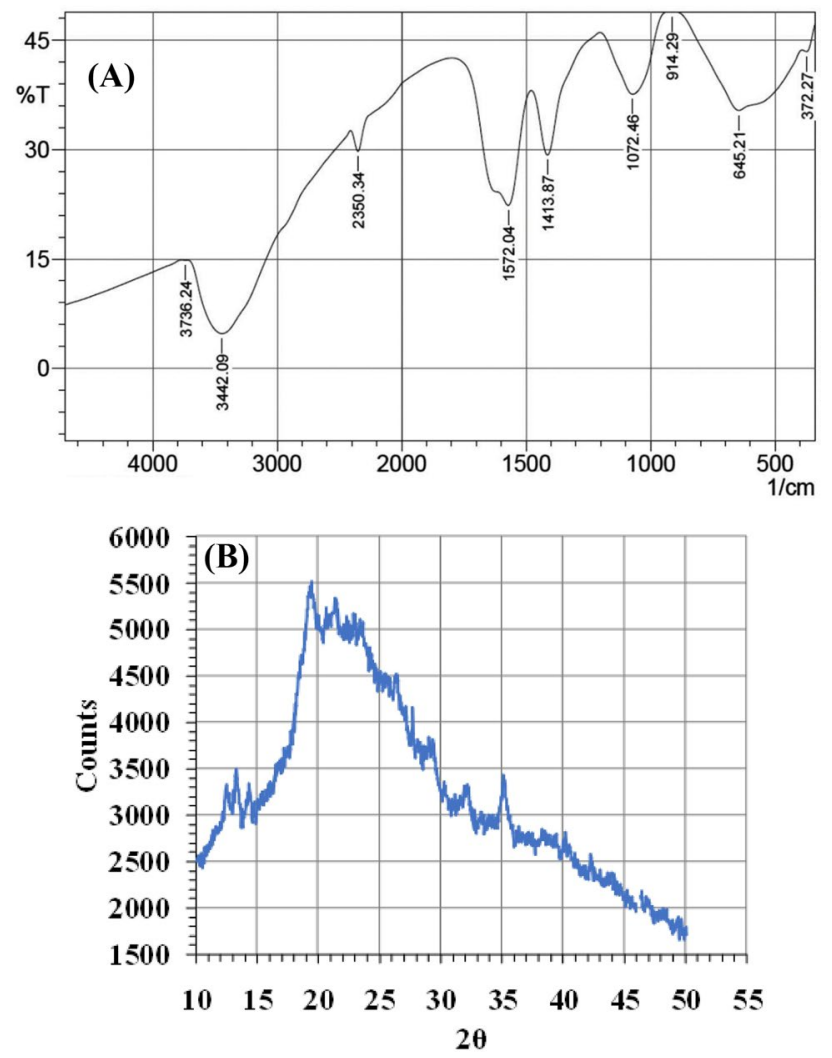

Figure 6. FTIR (A) and XRD (B) analyses of chitosan nanoparticles biosynthesized using Pelargonium graveolens leaf extract.

Thermal behavior of CNPs. The thermal behavior of CNPs was scrutinized by thermogravimetric analysis (TGA) in a temperature range from 30 to 800 . TGA is a thermal analysis that measures the modifications in physical and chemical features of a material such as CNPs as a function of constant changing heating rate. The change in the mass of CNPs was observed and analyzed with the change in temperature (Fig. 7A). The mass fluctuation of CNPs corresponds to the nature of chemical reactions. At the beginning of heating $\left(31.20-101.5^{\circ} \mathrm{C}\right)$, drying as a function of temperature can easily be seen as a quick initial mass dropping $(-23.118 \%)$. Then, the weight loss of CNPs showed multistage decomposition with increasing temperature but with lower weight losses. The lowest weight loss was recorded at a temperature range of $483.38-613.34{ }^{\circ} \mathrm{C}$, which was $2.461 \%$. Although CNPs have reasonable heat constancy, at the last temperature stage $\left(704.92-799.91^{\circ} \mathrm{C}\right)$, the nanoparticles kept a reasonable amount, i.e. $\sim 20 \%$, of their mass.

Investigation of the thermo analytical technique of differential scanning calorimetry (DSC) was applied to CNPs at different heating rates to determine the variation (positive or negative) in the amount of heat flow of CNPs as a function of temperature in the presence of a solvent reference. Both the CNPs and reference were sustained at the same temperature throughout the experiment. Thermo analytical information was gathered to create a phase diagram (Fig. 7B). As the thermodynamic system is changed, the CNPs undergo phase transitions, showing two definite broad endothermic peaks. The first broad endothermic peak was detected between 90 and $143{ }^{\circ} \mathrm{C}$ at $122{ }^{\circ} \mathrm{C}$, requiring a heat amount of $-358.87 \mathrm{~J} / \mathrm{g}$ CNPs. The other broad endothermic peak appeared at $242^{\circ} \mathrm{C}$, requiring $-64.18 \mathrm{~J} / \mathrm{g} \mathrm{CNPs}$. A single glass transition temperature of the CNPs was found at $180{ }^{\circ} \mathrm{C}$.

Isolation and identification of $\boldsymbol{B}$. cinerea. Following the isolation and purification trials, the most severe isolate was selected from several isolates based on the pathogenicity test. The colony characteristics of B. cinerea SIB-1 were observed on PDA plates, on which the fungus developed and sporulated on the culture medium to cover the majority of the $9-\mathrm{cm}$ plate during six days. The growth pattern on the plates showed the colonies as warty, fluffy, and appressed, grayish-white to light gray or dark gray. The conidia produced on the surface of the medium, in abundance all over the plates, can also be seen as concentric rings.

Data extracted from the SEM investigation (Fig. 8) show the conidial clusters carried on conidiophores and the branched mycelium, with conidiophores arising directly from the mycelium. They were more or less straight, monopodial branched toward the apex. The conidia were solitary and arranged in clusters or masses. The forms of the conidia observed were ellipsoidal and globose, and they were smooth, often with a slightly protuberant hilum and unicellular. The conidia measured $13.97 \pm 2.04 \mu \mathrm{m}$ in length and $9.53 \pm 1.45$ in width. 

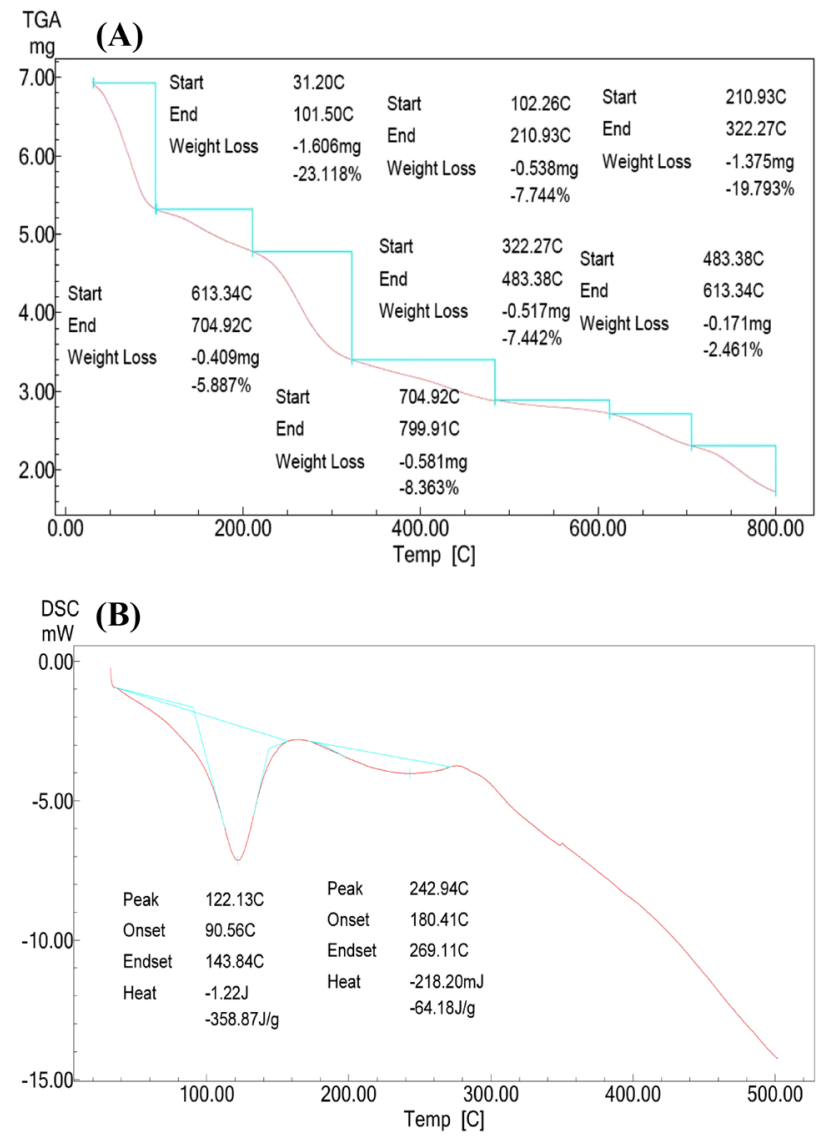

Figure 7. TGA (A) and DSC (B) analyses of chitosan nanoparticles biosynthesized using leaf extracts of Pelargonium graveolens.

Molecular identification. A 500 bp fragment (Fig. 9A) of the internal transcribed spacer (ITS) region was amplified from genomic DNA of $B$. cinerea isolate SIB-1 by using a specific primer. (Supplementary Firgure. S1) The amplicon was sequenced and compared to the NCBI public genome map using the BLAST program. Phylogenetic analysis using the neighbor-joining method was performed (Fig. 9B) to determine the relatedness between the sequenced gene and its homologs in other organisms. The created phylogeny is totally annotated and displays a tight correlation with those of comparable strains. Multiple nucleotide sequence alignment showed that the B. cinerea SIB-1 isolate was closely related to B. cinerea strain RGM 2565 (K934584). Accordingly, the current strain was banked in the NCBI GenBank with the accession number MZ570270.

Antifungal activity of CNPs. The in vitro antifungal activity of CNPs and chitosan (Fig. 10 and Table 4) revealed an obvious reduction in the growth of $B$. cinerea SIB-1 on potato dextrose agar plates, but the greatest fungal inhibition was the result of CNPs treatments. CNPs inhibited fungal growth by $73.58 \%$ at $1.0 \mathrm{mg} / \mathrm{mL}$, while chitosan showed only $31.16 \%$ growth inhibition under the same condition. In comparison, the recommended dose of Ridomil Gold (positive control) caused about $50 \%$ inhibition of the fungal growth.

To investigate the protective roles of CNPs against gray mold disease developed by B. cinerea SIB-1, different concentrations of the CNPs were tested on detached strawberry leaves (Fig. 11). The average percent of leaf area infected was determined for each concentration. In general, after five days, leaves treated with the CNPs had a lower disease severity compared with the control, which had a mean infected area of $85 \%$ with a severity class value of 4 . The lowest disease severity (class 0 ) was observed at the concentration of $25 \mathrm{mg} / \mathrm{mL}$, which only showed a $3 \%$ infected area. However, CNPs at 12.5 and $50 \mathrm{mg} / \mathrm{L}$ recorded leaf area infections of 27 and $23 \%$ with severity class values of 2 and 1, respectively. It was found that the disease dramatically progressed over time from one to five days on both the adaxial and abaxial sides.

\section{Discussion}

Nanotechnology has had a significant impact on several high-tech businesses in recent years, and it has been demonstrated that it has an impact on many microbial species, as well ${ }^{1}$.

There are several advantages of nanoparticles over the bulk form. Biologically, owing to the tiny size, nanoparticles straightforwardly penetrate and are easily taken up by the cell, which permits proficient accretion at the target site in the organism. Moreover, the retention of the nanoparticles at the target site has a longer clearance 


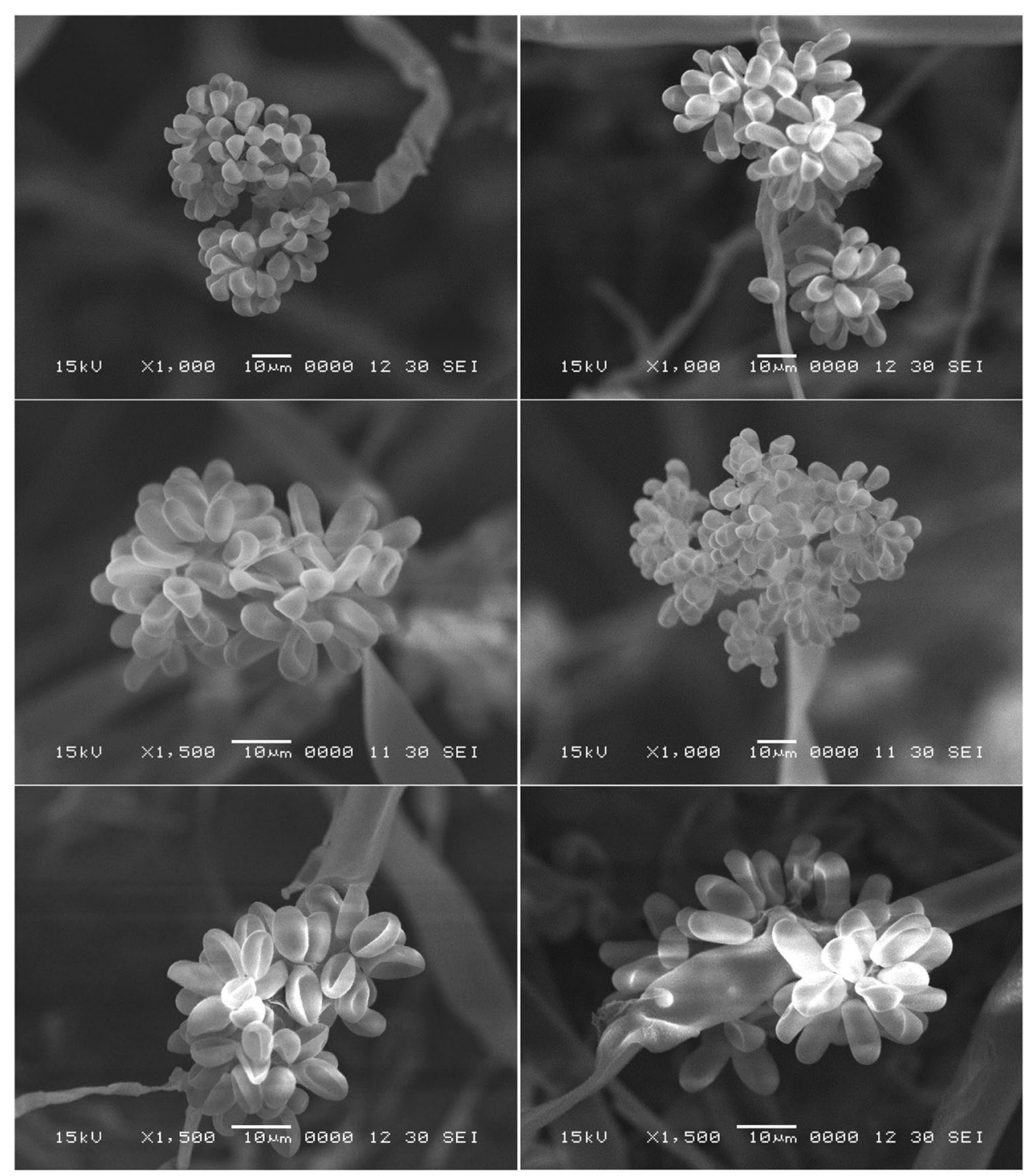

Figure 8. Scanning electron micrographs of B. cinerea SIB-1, showing conidial clusters carried on conidiophores at a magnification of $1000 \times$ and $1500 \times$ in different fields.

time, leading to an increase in therapeutic stability, bioavailability, and efficiency compared to the same dosage of the non-nanoparticulate form ${ }^{7,18}$.

Nanoparticles can be generated utilizing an assortment of strategies, including physical (ball milling, ultrathin films, spray pyrolysis, thermal evaporation, plasma arcing, lithographic procedures, pulsed laser desorption, layer-by-layer growth, sputter deposition, molecular beam epitasis, and diffusion flame synthesis of nanoparticles) and chemical (chemical solution deposition, electrodeposition, chemical vapor deposition, sol-gel process, soft chemical method, catalytic route, wet chemical procedure, hydrolysis coprecipitation, and Langmuir-Blodgett) methods, as well as hybrid techniques. These methods use high radiation and highly concentrated reductants and stabilizing agents that are destructive to human health and the environment as well ${ }^{7,18-20}$.

Alternatively, the biological-based procedure or green manufacture of nanoparticles is a bioreduction process with lower energy requirements. The technique is environmentally friendly and nontoxic, with greater stability, and nanoparticles are biosynthesized by applying a single-step process ${ }^{18}$. Moreover, to the best of the authors' knowledge, all phytofabrication of nanoparticles is mostly restricted to metal ions, and no previous reports on CNPs phytofabrication.Many examinations have demonstrated that plant extracts act as potential precursors for the biosynthesis of nanoparticles in nondangerous manners. Therefore, plants are utilized effectively and economically in the biosynthesis of several metal-nanoparticles ${ }^{7,10}$.

The universal procedure of metallic nanoparticle biosynthesis employs the plant as a bioreducing agent and metallic salt as a precursor, resulting in biocompatible and stable nanoparticles. This promising route of nanoparticle production using a biological system utilizes three main approaches, i.e., 1) the selection of solvent intermediate, 2) the choice of an ecological, benign reducing agent, and the choice of a nontoxic material as a 


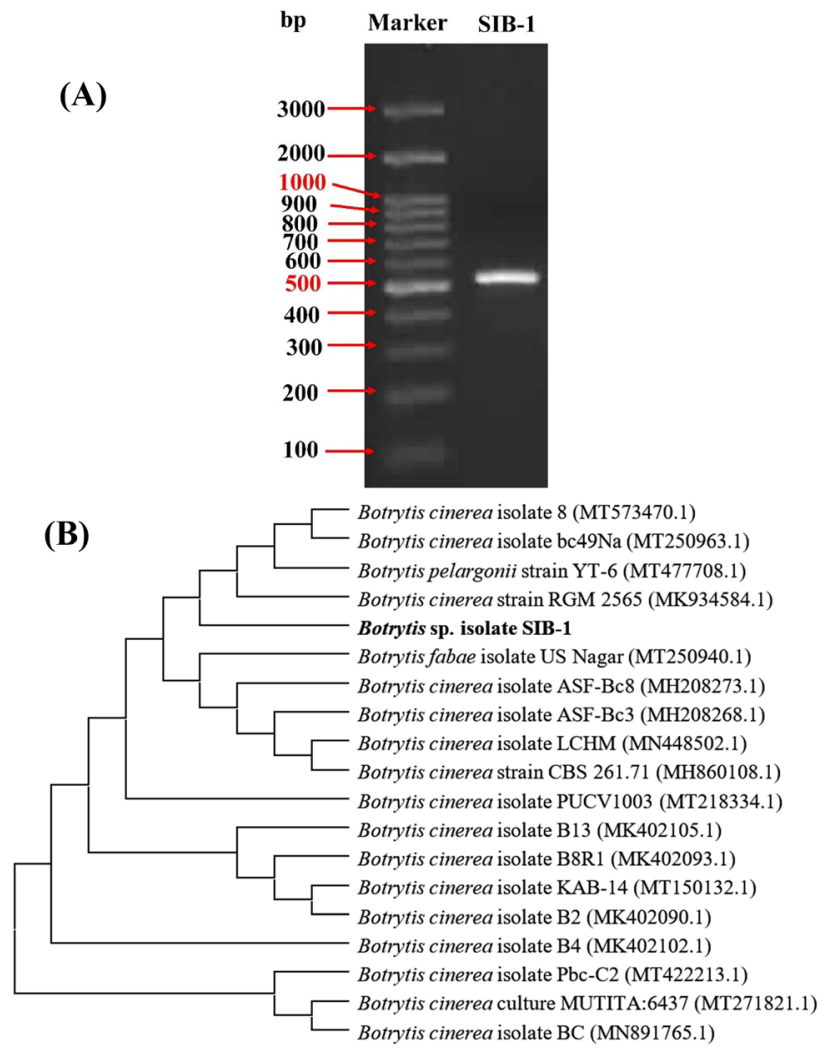

Figure 9. Profile of agarose gel electrophoresis of the PCR band of the PCR product of the amplified ITS fragment isolated from $B$. cinerea SIB-1 (A), and the evolutionary tree of the ITS gene's partial sequence of $B$. cinerea SIB-1 (accession number MZ570270), concerning the closely related sequences in GenBank (B).

capping agent to stabilize the biosynthesized nanomaterials ${ }^{21,22}$. Additionally, twelve well-known green chemistry principles have now become a reference guide for developing less hazardous chemical products ${ }^{23}$.

All previous approaches were found to be achieved in the selected plant (Pelargonium graveolens) and the transformed polymer (chitosan), additionally, the 12-green chemistry principles were strongly applied in the current work. To the best of the authors' knowledge, no previous work has reported on the synthesis of chitosan polymers into nanoforms using Pelargonium graveolens plants. Accordingly, the current paper describes a novel protocol for the green biosynthesis of CNPs, employing the leaf extract of Pelargonium graveolens. This procedure offers several merits over ordinary fabrication procedures.

Before proceeding to maximize the biosynthesis of CNPs, a primary characterization test based on UV/ visible spectra was applied to ensure the development of the nanoparticles. The current absorption peak wavelength was detected at $295 \mathrm{~nm}$; this result is in harmony with that previously reported at $285 \mathrm{~nm}^{24}$ and $320 \mathrm{~nm}$ in the UV region ${ }^{2}$. Compared with CNPs, the UV/visible spectrum of chitosan showed a wider absorption band intensity; therefore, the sharp intensity level of the CNP biopolymer indicates the success of the phytofabrication of $\mathrm{CNPs}^{2,24}$.

Exploring the experimental data of CNPs of the design matrix shows the highest level of CNPs located at the middle levels of the three tested variables, indicating the accuracy of both selected independent variables and their tested levels. It was obvious that the predicted values of CNPs were very adjacent to those of the trial CNPs; consequently, the residuals or error values were at their minimum, signifying another proven accuracy of the investigated parameters and their levels.

For the selection of the most appropriate model, the effect of each model term was screened at the $P$ level of 0.05. The tested terms (factors) that had lower $P$ values were considered significant and reliable for the modeling process. Regarding the model selection, $\mathrm{R}^{2}$ is considered a very important selection criterion; if $\mathrm{R}^{2}$ is higher than 0.9 , the regression model is defined as very significant, and the model is adequate; however, the $\mathrm{R}^{2}$ value should not be lower than $0.75^{25}$. The current quadratic model had high $\mathrm{R}^{2}$, adjusted $\mathrm{R}^{2}$, and predicted $\mathrm{R}^{2}$ values, which were very close to one. Consequently, the quadratic model was the best-fitted model.

$\mathrm{R}^{2}$ is defined as the amount of change in the observed (experimental) response (CNPs) that is described by the three tested factors. Generally, $\mathrm{R}^{2}$ can help choose the best-fitted model. All types of $\mathrm{R}^{2}$ range from zero to 1 . The closer to 1, the better the modeling of the experimental data. Interestingly, increasing the number of factors (predictors) leads to a continuous increase in the $\mathrm{R}^{2}$ value, irrespective of the significance of the factors. Therefore, the adjusted $\mathrm{R}^{2}$ is an improved $\mathrm{R}^{2}$ that considers the number of factors (variables) in the model. Contrary to $\mathrm{R}^{2}$, the adjusted $\mathrm{R}^{2}$ may be reduced with the addition of extra terms (factors) to the model. Therefore, the adjusted 


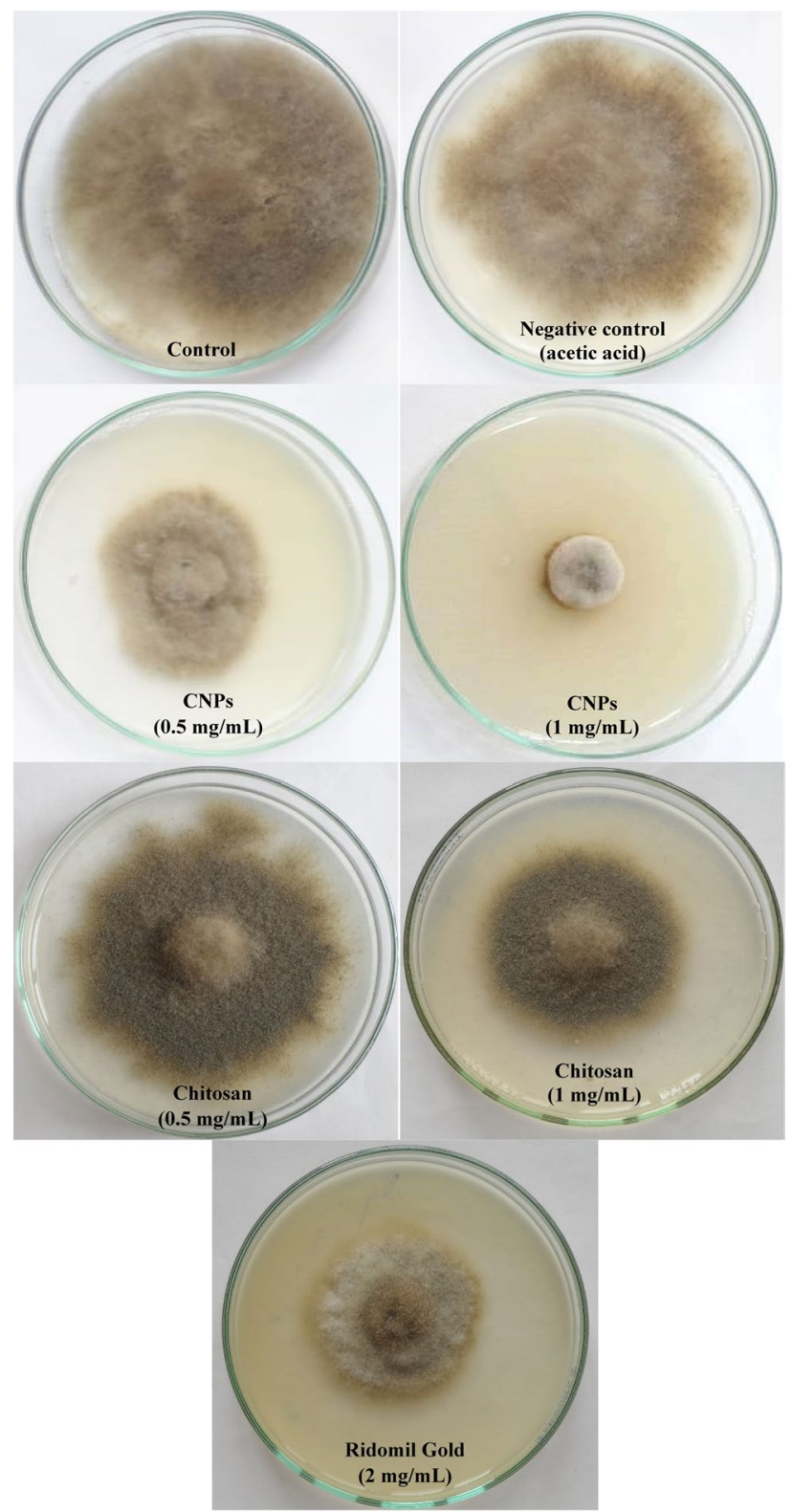

Figure 10. Antifungal activity of CNPs produced using Pelargonium graveolens leaf extract against $B$. cinerea SIB-1.

\begin{tabular}{|l|l|l|}
\hline \multirow{2}{*}{ Concentrations $(\mathbf{m g} / \mathbf{m L})$} & \multicolumn{2}{|l|}{ Inhibition of fungal growth, \% } \\
\cline { 2 - 3 } & CNPs & \multicolumn{2}{|l|}{ Chitosan } \\
\hline 0.0 & $0.0 \pm 0.00^{\mathrm{e}}$ & $11.25 \pm 2.75^{\mathrm{d}}$ \\
\hline 0.5 & $44.15 \pm 3.43^{\mathrm{b}}$ & $31.16 \pm 3.89^{\mathrm{c}}$ \\
\hline 1.0 & $73.58 \pm 0.75^{\mathrm{a}}$ & \multicolumn{2}{|l}{} \\
\hline
\end{tabular}

Table 4. Percentage of mycelia inhibition of $B$. cinerea SIB- 1 by CNPs and chitosan after 10 days of incubation at $28{ }^{\circ} \mathrm{C}$. Means followed by the same letter are not significantly different based on Duncan's multiple range test at $5 \%$. 


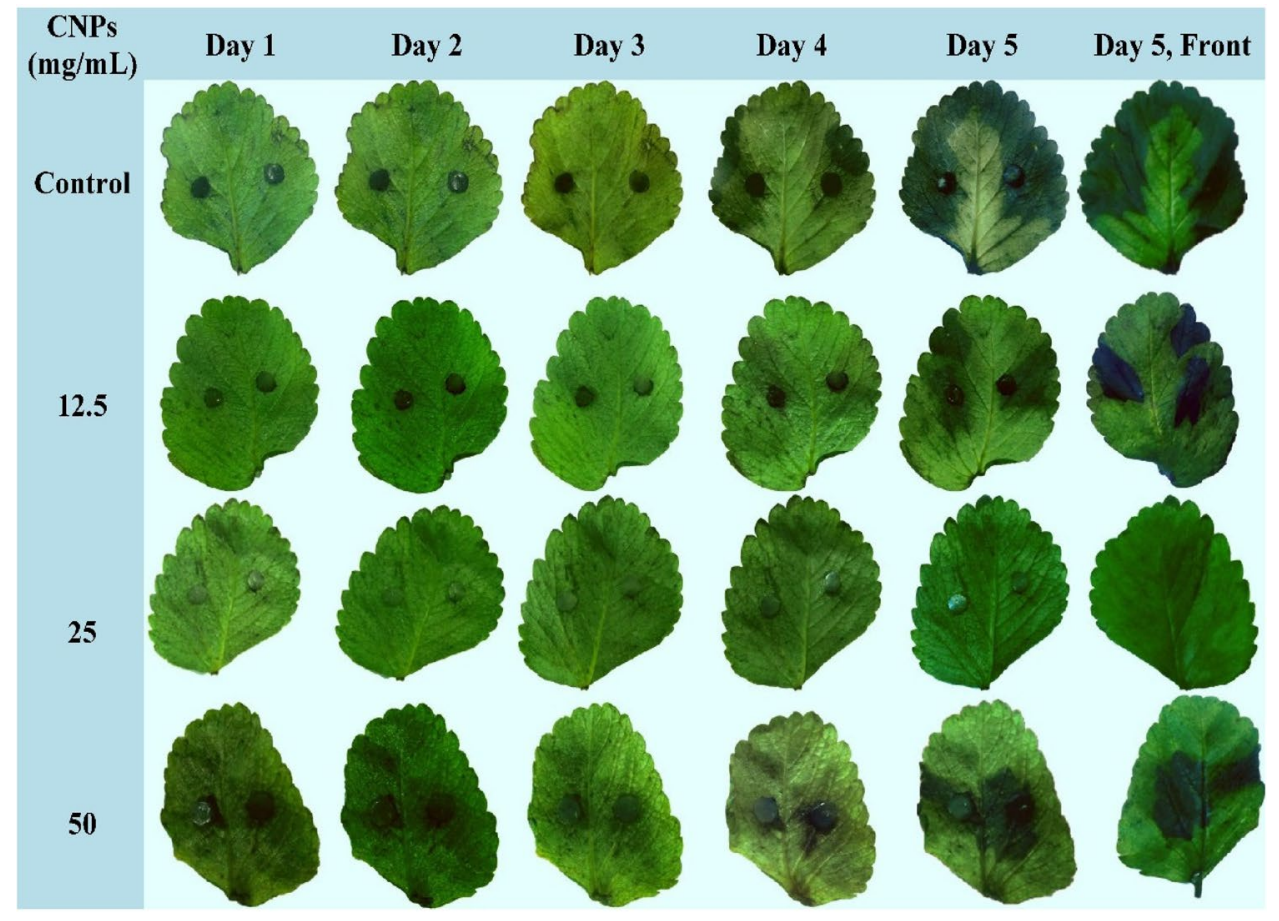

Figure 11. The protective effect of chitosan nanoparticles against gray mold disease caused by B. cinerea SIB-1 on detached strawberry leaves using different CNPs concentrations.

$\mathrm{R}^{2}$ is a better indicator than $\mathrm{R}^{2}$ to judge how well the model fits the data. The predicted $\mathrm{R}^{2}$ is used to determine the degree of the predictive capability of the model, e.g., to predict the value of the CNP response at new levels of the tested factors. Moreover, it is more beneficial than the adjusted $\mathrm{R}^{2}$ for comparing and selecting the models. Therefore, the quadratic model was selected as a modeling base in CNP bioprocessing.

The BBD data were subjected to ANOVA. The model exhibited a high $F$-value and low $P$-value; additionally, the lack-of-fit was not nonsignificant, indicating the significance of the proposed overall model. Moreover, the ratio of adequate precision is higher than 4 , which is a suitable indicator that this model can be successfully employed to work within the tested range of the various tested factors along with the design space to maximize CNP biosynthesis. Another precision and trustiness of the experimental design can be noted by the lower value of the C.V. and greater value of adequate precision, which are desirable for the reliability of the model.

The weight of every individual factor was diagnosed, and the $P$-value was again utilized. However, the values of $P$ indicate that the model terms are significant $(<0.05)$, indicating that they are important phytofabrication parameters of CNPs. This also suggests that the variables and their established levels, as well as the investigational design, are well defined and attain the peak performance of CNP phytofabrication. Therefore, the projection model was created based on such proven terms.

Data of ANOVA displays that the predicted $\mathrm{R}^{2}$ and adjusted $\mathrm{R}^{2}$ are close to each other. The values of both kinds of $\mathrm{R}^{2}$ should be less than $20 \%$ of each other to be in decent agreement ${ }^{25}$. In the current investigation, the predicted $\mathrm{R}^{2}$ was in harmony with the adjusted $\mathrm{R}^{2}$ value, indicating high compatibility between the predicted and experimental values of CNP biosynthesis and indicating the satisfactory predictive ability of the model within the examined range.

Some of the model terms showed a negative coefficient estimate value, which indicates that such a variable has an antagonistic effect on CNP biosynthesis by . graveolens at higher concentrations. The positive coefficient value, on the other hand, indicates a cooperative effect, and the variable(s) increase CNP biosynthesis with the continuous increment of the level of the investigated factor within the region of the experiment.

Model adequacy was checked. The normal plot of residuals was plotted to check the externally studentized residuals versus normal probability (\%). Values show an equal distribution of the residual data, indicating that the variance of CNP biosynthesis was independent of the biosynthesis process, thus supporting the adequacy of the model. Moreover, residuals were found to be very low at all tested points. This implies that the model can fit the actual experimental data faithfully. Additionally, the model prediction points vis the actual points lie much nearer to the line of perfect prediction. Thus, the model has a significant generalization capacity for CNP biosynthesis. Moreover, the Box-Cox plot of the model transformation of chitosan nanoparticle biosynthesis using $P$. graveolens leaf extract confirms the suitability of the design and data. The value of 1 concluded no recommendation for data transformation in this model. Consequently, these two adequacy tests authorize the aptness of the design and obtained data. Model adequacy was checked by plotting the normal probability of the externally studentized residuals. Most of the data points aggregated thoroughly around the straight line and were, thus, considered normally distributed without linearity. No value is located away from the general mean since the 
extreme residual values on both sides are not wanted. The plot of predicted vis actual values. The pattern also displays a normal distribution, supporting the adequacy of the model.

Analysis of the 3D plots demonstrates that all the pair tested factors generated a peak of CNP biosynthesis around the center point of the design space, meaning that the tested ranges of the three factors were carefully selected, and the model best fit the design.

To find out the best-predicted combinations that maximize CNPs, the desirability function was used, whose value ranges from undesirable (zero) to desirable (one). As the response approaches the goal, the desirability value becomes closer to 1 . The desirability value is generally estimated as a mathematical evaluation of the optimization process before experimental validation ${ }^{26}$. Accordingly, the optimum levels of the incubation period, temperature, and initial chitosan concentration that maximize CNP biosynthesis by P. graveolens were estimated by solving the prediction equation. Among several options, the best solution was selected based on the desirability value. The current desirability value was sufficiently high since it reached the peak that validate the optimization process. Then, the predicted amount of CNPs was estimated, which was found to have a high intensity of agreement between the experimental values, suggesting that the desirability function effectively ascertains the best-predicted situations for the green synthesis of CNPs by using P. graveolens leaf extract.

The theoretical estimation of the polynomial model is an estimate based on a reasonably studied area of the tested independent variables; therefore, the guarantee of the real prediction effectiveness of the equation under real conditions is critical. However, the theoretical value of CNP biosynthesis was valid, since it showed close similarity to that of the experimental one. That is, in turn, produces strong evidence for the fitness of the design and the modeling process, utilizing the tested ranges of the studied variables.

Following the optimization conditions of the biofabrication process of CNPs from chitosan by P. graveolens leaf extract, the surface morphological structure of the obtained CNPs was monitored by SEM, which are widely considered the main accepted procedures for the characterization of nanoparticles. These techniques are erroneously used interchangeably, but in reality, they vary substantially. However, both techniques provide some similarities but a distinct analysis, which is why the accurate interpretation of their images is essential. Collectively, SEM and TEM offer powerful tools for the investigation of size, shape, surface area, crystal structure, and morphological structure. Although TEM systems can bring much greater 2D resolution for size analysis, SEM provides accurate information about the 3D surface and shape features ${ }^{27,28}$.

The 3D SEM image of CNPs exhibits a good dispersion of the nanoparticles, which are entangled to form a larger exposed surface area, making the CNPs very appropriate for adsorption ${ }^{29}$. Like the current phytofabricated CNPs, most of the CNPs prepared from chitosan were spherical in shape ${ }^{30}$, and few had oval pleated ${ }^{31}$ or rod-shaped structure ${ }^{29,32}$.

The 2D TEM image undoubtedly indicates that the CNPs show a highly porous surface owing to low agglomeration attributes. These porous and agglomerated CNPs have been considered key phenomena for the synthesis of novel CNPs, hence maximizing their usefulness as antibiological phytosynthesized nanomaterials in biomedical and agricultural applications, where the porous nature can effectively adsorb harmful chemicals and antagonize the pathogens ${ }^{2,33}$. In contrast to bulk materials, which have lower porosity, nanoparticles with high porosity have a greater specific exterior area and high reaction activity ${ }^{1}$.

The terms agglomeration and aggregation are repeatedly used interchangeably, but they definitely differ, where agglomeration indicates more weakly bonded particles and aggregation indicates strongly bonded or fused particles. In our case, the CNPs showed low agglomeration without aggregation, and the low agglomeration phenomenon is accepted since many nanoparticle types have high ionic strength and agglomerate in aqueous matrices, such as in phosphate-buffered saline and cell culture medium ${ }^{34,35}$.

EDXS study was used together with electron microscopy investigation to analyze the component elements of CNPs. When the electron beam of SEM hits the inner shell of an element atom, its inner-shell electron is relocated by another electron from an outer shell to fill the vacancy, and the process is accompanied by the release of an energy difference in the form of an X-ray that is unique to the specific element. Moreover, the intensity of the specific X-ray is directly related to the concentration of the element in the particles ${ }^{36}$. However, the test confirms the presence of the various elemental compositions of native chitosan, confirming the uniformity and stability of CNPs during the biotransformation process.

The $\zeta$-potential is an indicator of the stability of colloidal dispersions. The weight of the $\zeta$-potential specifies the degree of electrostatic repulsion among similarly charged adjacent particles ${ }^{37}$. For tiny particles and molecules, a high $\zeta$-potential confers stability and resists aggregation of nanoparticles in the solution or dispersion. In contrast, at small $\zeta$-potentials, attractive forces may exceed, leading to flocculation owing to the breakdown of the dispersion. Therefore, colloids with high negative or positive $\zeta$-potentials are more electrically stabilized than those with low $\zeta$-potentials, which tend to coagulate or flocculate ${ }^{38}$. The current $\zeta$-potential value of CNPs suggests nanoparticles with good stability. The CNPs were positively charged. From an antimicrobial point of view, when $\zeta$-potential is positive, particles can easily interact with the negatively charged cell membrane and/ or DNA of a biological system and can then be released simply into the cytoplasm of the cell ${ }^{39}$.

Regarding FTIR, the presence of various intense bands indicates the presence of a capping agent, which acts as a stabilizer that inhibits the overgrowth of nanoparticles and prevents their aggregation and/or coagulation in colloidal synthesis. Therefore, the observed intense bands were matched with standard values to classify the functional groups. The first range of bands that appeared in the spectra was due to stretching vibrations of $\mathrm{OH}$ groups at wavenumbers ranging from 3736 to $3442 \mathrm{~cm}^{-1}$, indicating the presence of alcohols and phenols. The stretching vibration of methylene $(\mathrm{C}=\mathrm{H})$ was at $2350 \mathrm{~cm}^{-1}$. It is also already known that the band at $2350 \mathrm{~cm}^{-1}$ generally arises from the background $\mathrm{CO}_{2}{ }^{40}$ and has no corresponding group associated with the chitosan structure. Absorption at wavenumber 1572 was correlated to the vibrations of carbonyl bonds $(\mathrm{C}=\mathrm{O}$ stretching) of the amide group CONHR or protonated amine $\left(\mathrm{NH}_{2}\right.$. Bending vibrations of the methyl group $(\mathrm{C}-\mathrm{H}$ bending, alkane) of CNPs were visible at $1413 \mathrm{~cm}^{-1}$. Absorption in the wavenumber range 1072 and $914 \mathrm{~cm}^{-1}$ is generated 
from the stretch vibration of $\mathrm{CO}$ groups $(\mathrm{COH}$ and $\mathrm{COC})$ in the oxygen bridge, emerging from the deacetylation of chitosan. At the end of the FTIR spectra, the small peak at $645 \mathrm{~cm}^{-1}$ corresponds to the wagging of the saccharide structure of chitosan ${ }^{41}$. FTIR analysis strongly emphasizes the structural stability of chitosan during phytoconversion into CNPs.

XRD analysis is a fast practice, primarily used in materials science for the phase identification of a crystalline nature and can deliver information on unit cell dimensions; thus, the XRD pattern is considered the fingerprint of periodic atomic arrangements in a given material ${ }^{32}$. The XRD of the current CNPs showed three peaks at 2-theta of 13,19 , and $35^{\circ}$. It is conventionally accepted that chitosan stretches two characteristic peaks at 2-theta of 10 and $20^{\circ}$, and the current shift that occurred to CNPs from the normal chitosan peaks indicates the amplified amorphous nature, thus lessening the crystal structure of chitosan, which comes in line with studies that focused on decreasing the crystallinity for improving the sorption properties of the materials ${ }^{32,42}$.

Both TGA and DSC investigations were performed, and both are measures of the thermo analytical features used to describe the analysis of nanoparticles that take part in chemical reactions over a controlled temperature range. TGA measures the differential thermal analysis in terms of the change in mass of the sample in relation to temperature changes or as a response of time with constant temperature and/or constant mass loss. DSC, on the other hand, measures the heat flow released or required against the temperature change at a particular time. The main dissimilarity between TGA and DSC is the method of measuring the changes in samples that are triggered by heat ${ }^{29,31,32,43}$.

At the beginning of TGA, common drying as a function of temperature can easily cause a quick initial drop in CNP mass due to the loss of residual water bound to the two polar groups in CNPs, which is not known to correspond to any chemical reactions ${ }^{32}$. Another reason for the drop in weight at the beginning step may be due to the dehydration of the saccharide rings, depolymerization, and decomposition of volatile products ${ }^{29}$. Next, successive weight losses in CNPs with increasing temperature may be due to evaporation and/or sublimation; however, multistage decomposition shown as a step-like pattern is due to the thermal degradation of CNPs ${ }^{43}$.

The loss of weight in the next stages of thermal analysis may be due to the decomposition of the polymer matrix; however, the CNPs did not fully decompose at the high temperature $\left(800^{\circ} \mathrm{C}\right)$ and conversely showed some stability in the polysaccharide structure. This result indicates that CNPs are thermally stable over a temperature up to $800^{\circ} \mathrm{C}$, which may be due to the high crosslinking of the CNPs that forms a stronger and stiffer hydrogel network ${ }^{29,32,43}$.

However, the thermal analysis steps were not blended during dynamic TGA; however, there is still a possibility of hidden interference of the decomposition steps, necessitating either far slower heating rates or stepwise TGA methods. That is why TGA itself may not be sufficient to identify the decomposition products, therefore chemical testing such as DSC is often, required alongside TGA, to ascertain the identities of suspected decomposition products ${ }^{31}$

DSC of CNPs was performed to highlight phase transitions and clarify every single step of the thermal degradation mechanism. Usually, the temperature program for a DSC analysis is designed such that the sample holder temperature increases linearly as a function of time. That, in turn, can offer pieces of evidence about physical phenomena, such as glass transition, thermal stability, and purity ${ }^{31}$. Two definite broad endothermic peaks were generated, the first at a lower temperature that was due to the removal of absorbed water. The second endothermic peak that appeared at $242^{\circ} \mathrm{C}$ is generally associated with the breakage of cross-linkage of CNPs. Additionally, the higher value of the glass transition temperature is due to the presence of a crosslinking agent and high thermal stability. Only a single glass transition in the DSC heating curves indicates the uniformity of the CNPs under high temperatures ${ }^{29}$. The transformation of chitosan into CNPs decreases the crystallinity due to changes in the solid-state structure of chitosan due to crosslinking, and thus, the decomposition of CNPs occurs above $300^{\circ} \mathrm{C}^{32}$.

Interestingly, in the present study, all proceeding investigations came in harmony with each other to provide an accurate perspective for the characterization of CNPs. Moreover, the currently proposed phytofabrication method for CNP preparation is considered ideal for generating high-quality CNPs.

The developed phytosynthesized CNPs were monitored regarding their antifungal properties. Depending on the molecular weight, concentration, degree of substitution, and the type of functional groups on chitosan, the free chitosan polymer exhibits various antifungal activities against a wide array of fungi. Derivatives of the polymer can be formed to target specific pathogens. Chitosan shows natural antifungal capability without the necessity for any chemical alterations ${ }^{2}$, which is why the generated CNPs were tested against the isolated $B$. cinerea SIB-1.

Koch's postulates were applied to the isolated fungi to confirm the pathogenic ability and to select the most aggressive isolate as well. The colony characteristics of the isolated fungus and SEM investigation showed the typical features of the already known phytopathogenic B. cinerea SIB-1. These features are in line with those previously described ${ }^{17}$.

A severe phytopathogen (Botrytis cinerea SIB-1) was used in this study as a model for the evaluation of CNPs as an anti-biological agent. The main reason for selecting such phytopathogen is the wide host range since it can infect more than 200 host plants, and it can infect several parts of the plants, including the upper parts such as seeds, leaves, bulbs, and other propagation material at pre- and postharvest stages. Moreover, Botrytis spp. infect the host plant in all climate areas of the world and under great humidity in the presence or absence of water films. The fungus can generate high numbers of conidia that pose a long-lasting threat to susceptible hosts; in addition, the genotypic and phenotypic variation of the fungus is another broad-spectrum thread for the plant production sector ${ }^{16,17}$. Importantly, the fast alterations in populations and resistance in response to exposure to xenobiotics, e.g., fungicides, are quite widespread in the genus ${ }^{44}$, urging the discovery of alternative commercial approaches of considerable disease suppression to be integrated into crop management protocols ${ }^{15}$.

The selected fungus was identified as $B$. cinerea SIB- 1 on a molecular basis, which is sensitive and specific for the rapid recognition of filamentous fungi at different systematic levels. The ITS region is usually used and 
can be adequate for fungal identification at the species level. The ITS region is also contemplated among the markers with the fastest and uppermost probability of precise identifications for a very broad group of fungi ${ }^{45}$. Interestingly, the culture morphology, SEM investigation, and molecular identification computably confirmed the fungus to be $B$. cinerea SIB-1.

Gray mold caused by the phytopathogenic Botrytis cinerea, is a serious disease that affects all strawberry growing regions and is the main cause of concern most years. The gray mold disease is a problem not only in the field, but also during storage, transportation, and marketing of strawberries as a result of severe rot as the fruits begin to ripen. Leafs, fruit caps, flower stalks, petals, and crowns are among the other parts infected by the fungus. CNPs showed strong inhibition against $B$. cinerea SIB-1. Possible protocols include inhibition of mycelial growth and sporogenesis ${ }^{3}$.

There are three mechanisms proposed as the inhibition mode of chitosan. In the first mechanism, the cell membrane of fungi is the main target of chitosan. The inhibitory effect of chitosan may be related to its interaction with the cell membrane of the fungal cell and alteration of membrane permeability ${ }^{46}$. The positive charge of chitosan allows it to interact with phospholipid components of the fungal cell membrane that are negatively charged. This increases membrane permeability, allowing cellular contents to leak out, ultimately resulting in cell death. The second mechanism involves chitosan acting as a chelating agent, binding to trace elements and rendering them unavailable to fungi for normal growth. Finally, the third mechanism proposed that chitosan could pass through fungi's cell walls and bind to their DNA or proteins. This will stop the production of essential proteins and enzymes by inhibiting the synthesis of $\mathrm{mRNA}^{3}$. Chitosan inhibited mycelia growth, sporulation, and spore germination. It induces morphological changes characterized by excessive branching, mycelial swelling, agglomeration of hyphae, abnormal shapes, dissolution of protoplasm, large vesicles, cytoplasm aggregation, or empty cells devoid of cytoplasm in the mycelium ${ }^{47}$.

Ridomil Gold is a systematic fungicide that inhibits fungal development by interfering with the biosynthesis of sterols in the cell membrane. Thus, provides excellent disease control, ensures double protection to the target plants due to systemic activity of Mefenoxam fungicide and contact protective activity of mancozeb fungicide. Mefenoxam penetrates rapidly into the plant through the leaves and stems and is distributed upwards with the flow of sap. This way, new growth is protected as well. Mancozeb provides a protective film on the surface of the plant and inhibits germination of the spores and controls leaf and tuber blight as well as leaf spot disease.

The current experimental results on strawberry leaves show that treatment with CNPs reduced infection lesions. A key factor for a pathogenic fungus to be able to magnificently infect plants is to secrete a category of effector proteins into plant cells, which makes plants more susceptible to diseases ${ }^{48}$. These effector proteins decreased after treatment with chitosan ${ }^{5}$; moreover, chitosan can stimulate defense-related enzymes and augment the accumulation of antimicrobial ingredients in the infected plant, mainly diminishing the success rate of the fungal infection and inducing plant resistance ${ }^{49}$.

It is of essential importance to note that leaf treatment with a high concentration of CNPs $(50 \mathrm{mg} / \mathrm{mL})$ was found to have a reverse effect on the infected area compared with those of lower concentrations. These results suggested that increasing the concentration of nanoparticles might result in the crowding of these particles on the leaf pores that limit their penetration into the inner tissues. Furthermore, several findings concluded the potential phytotoxicity of these nanoparticles at high concentrations. In this respect, the application of CNPs at a concentration higher than the optimum causes a reduction in the mineral and nitrogen contents of the coffee leaves ${ }^{50}$. Similarly, high concentrations of chitosan nanoparticles markedly reduce the growth and development of Capsicum annuum, while lower concentrations have a growth-promoting effect ${ }^{51}$. These results suggest additional investigation on the optimum concentration of CNPs and further indicate the need for caution when using CNPs to reduce the harmful side effects of elevated concentrations.

\section{Materials and methods \\ Formulation of plant extract. Fresh leaves of Pelargonium graveolens were collected from the El-Natrun area ( $30^{\circ} 22^{\prime} 39^{\prime \prime} \mathrm{N}$ latitude and $30^{\circ} 21^{\prime} 1.08^{\prime \prime}$ E longitude), Northern West Nile Delta, 62 miles from Cairo, Egypt. The leaves were washed thoroughly three times with tap water and then with distilled water to eliminate any impurities. Finally, $25 \mathrm{~g}$ of thoroughly washed and finely cut leaves were dipped into a conical flask containing $100 \mathrm{~mL}$ of distilled water, mixed, and boiled for $10 \mathrm{~min}$. After boiling, the solution was then filtered through filter paper (Whatman No. 1). The filtered extract of Pelargonium graveolens was collected and used for the bio- synthesis of CNPs.}

Phyto-synthesis procedure of CNPs. Chitosan, obtained from Bio Basic Inc., Toronto, Canada, was liquefied at $1 \%(\mathrm{w} / \mathrm{v})$ with acetic acid $(1 \%, \mathrm{v} / \mathrm{v})$, and the $\mathrm{pH}$ was raised to 5 with $1 \mathrm{~N} \mathrm{NaOH}$ and kept under magnetic stirring for $24 \mathrm{~h}$. Equal amounts $(10 \mathrm{~mL})$ from each of the plant extracts and chitosan solution were blended and incubated at $50{ }^{\circ} \mathrm{C}$ under shaking for $30 \mathrm{~min}$. After incubation, the turbidity was centrifuged at $10,000 \times g$ for $10 \mathrm{~min}$. To remove the unreacted chitosan from the produced nanoparticles, it was washed several times with acetic acid solution. The precipitate was then obtained by centrifugation of the mixture at $10,000 \times g$ for $10 \mathrm{~min}$. The resultant CNPs were then freeze-dried. The resultant CNPs were redissolved again in $1 \%$ acetic acid, and the UV/visible absorbance spectrum of the prepared CNPs was determined by a double beam spectrophotometer at $295 \mathrm{~nm}$. A standard calibration curve prepared from known concentrations of CNPs was prepared in 1\% acetic acid at various serial dilutions and was used to estimate the final concentration of CNPs $(\mathrm{mg} / \mathrm{mL})$.

UV-Visible spectrum. The generated CNPs were monitored by detecting the peak absorbance at an array between 200-400 $\mathrm{nm}$ utilizing an Optizen Pop-UV/Vis spectrophotometer. 
Optimization of CNPs biosynthesis by BBD. The matrix of BBD was generated to establish the best levels of the chosen process variables affecting chitosan nanoparticle biosynthesis using $P$. graveolens leaf extract and to study the individual and interaction effects between these variables. The factors were incubation period $\left(\mathrm{X}_{1}, 30-90 \mathrm{~min}\right)$, temperature $\left(\mathrm{X}_{2}, 40-60{ }^{\circ} \mathrm{C}\right)$, and chitosan concentration $\left(\mathrm{X}_{3}, 0.5-1.5 \%\right)$. Each process variable ranges at three levels $(-1,0,+1)$, with five central points, resulting in a total of seventeen trials (Table 1$)$. The linear, interaction, and quadratic influences of the three selected process variables affecting chitosan nanoparticle biosynthesis were determined to explore the relationship between chitosan nanoparticle biosynthesis $(Y)$ and the optimum level of each of the tested variables. The next second-order polynomial function was applied:

$$
Y=\beta_{0}+\sum_{i} \beta_{i} X_{i}+\sum_{i i} \beta_{i i} X_{i}^{2}+\sum_{i j} \beta_{i j} X_{i} X_{j}
$$

where $Y$ is CNP biosynthesis using $P$. graveolens leaf extract, $X_{i}$ is the process variable at the coded levels, $\beta_{0}$ is the regression coefficient, $\beta_{i}$ is the linear coefficient, $\beta_{i j}$ is the interaction coefficient and $\beta_{i i}$ is the quadratic coefficient.

The investigational design and statistical examination were conducted using the Windows edition of DesignExpert software (version 12, Stat-Ease, Minneapolis, USA). The STATISTICA version 8 program was used to draw 3D surface plots.

Characterization of CNPs. SEM investigation. The size, construction, and morphology of the CNP samples were examined. After coating by a gold sputter coater (SPI-Module), samples were examined by SEM (model JEOL-JSM-IT200) at $20 \mathrm{kV}$ at the Electron Microscope Unit, Faculty of Science, Alexandria University, Alexandria, Egypt.

TEM investigation. Another morphology investigation of CNPs was carried out by TEM. The samples were examined with a transmission electron microscope unit (TEM; JEM-2100 Plus, JEOL Ltd., Japan) at the Central Laboratory, City of Scientific Research and Technological Applications, Alexandria, Egypt.

EDXS examination. EDXS is commonly used to determine the elemental composition and characterization of a sample. The electron beam focused on a single nanoparticle by TEM through the program functions to obtain insight information about the CNPs under observation.

$\zeta$-potential of the synthesized CNPs. The zeta $(\zeta)$ potential determines the surface charge properties of the chitosan nanoparticles. The $\zeta$-potential of the CNPs was quantified utilizing Malvern analytical Zetasizer software Ver. 7.13 equipped with a laser Doppler and detected with phase analysis light scattering. The measurements were performed at $25^{\circ} \mathrm{C}$, taking samples in the liquid state ${ }^{52}$.

FTIR spectroscopy analysis. FTIR spectroscopy assessment was conducted to analyze the surface properties of the CNPs, which were ground with $\mathrm{KBr}$ pellets used for FTIR measurements. The FTIR spectrum of CNPS was measured using a Shimadzu FTIR-8400 S spectrophotometer in the range of $4500-500 \mathrm{~cm}^{-1}$ at a resolution of $1 \mathrm{~cm}^{-1}$.

XRD pattern. XRD is a crucial technique for determining the structural properties of nanoparticles. The XRD patterns were recorded using a diffractometer (Bruker D2 Phaser 2nd Gen). The X-ray source was operated with a Cu anode with a voltage of $30 \mathrm{kV}$ and a current of $10 \mathrm{~mA}$. Diffraction intensity was measured at $25.7^{\circ} \mathrm{C}$ and a scanning rate of $2 \% \mathrm{~min}$ for $2 \theta=10-50^{53}$.

TGA of CNPs. The CNP sample was withered at $60^{\circ} \mathrm{C}$ for $1 \mathrm{~h}$ and mounted in a platinum sample pan. TGA was accomplished using a thermoanalyzer of type $50-\mathrm{H}$. TGA was operated in the range from 25 to $800^{\circ} \mathrm{C}$, with a $20 \mathrm{~mL} \mathrm{~min}^{-1}$ flow rate, under a nitrogen atmosphere at an increment of $10{ }^{\circ} \mathrm{C} \mathrm{min}{ }^{-1}$. The chart was drawn as temperature versus weight loss percentage.

DSC analysis. DSC (60-A) was used to assess the CNP pyrolysis pattern. The CNP sample was dried at $60{ }^{\circ} \mathrm{C}$ for $1 \mathrm{~h}$ and mounted in an aluminum sample pan. The examination was operated under nitrogen atmosphere conditions with a heating rate of $10{ }^{\circ} \mathrm{C} \mathrm{min}{ }^{-1}$ and a flow rate of $30 \mathrm{~mL} \mathrm{~min}{ }^{-1}$. The thermogram was generated between 25 to $500{ }^{\circ} \mathrm{C}$. The initial breakdown temperature for the CNPs, as presented by thermogravimetric analysis, was chosen for the DSC upper limit. The graph was mapped as heat flow versus temperature.

Isolation and identification of the fungal pathogen. The model fungus used in this study was B. cinerea, a causative agent of gray mold. The fungus was isolated from blighted fruits of strawberry (Fragaria virginiana, var. Fortuna) collected from open fields in the Menoufia government ( $30^{\circ} 18^{\prime} 02^{\prime \prime} \mathrm{N}$ latitude $31^{\circ} 00^{\prime} 30^{\prime \prime}$ E longitude) during March 2021. The collected samples were kept at $25^{\circ} \mathrm{C}$ and $90 \%$ relative humidity for 4 days in a glass box to facilitate fungal sporulation. The purified culture was obtained by a single-spore technique on PDA medium, purified, and then stored $\left(4^{\circ} \mathrm{C}\right)$ until further use. Primary morphological identification was carried out with the aid of the Miclea et al. ${ }^{17}$ method. One pathogenic isolate of B. cinerea SIB-1 was selected based on the pathogenicity test. The most severe isolate was selected, and further confirmational identification methods were used. The morphology of the Botrytis cinerea SIB-1 was examined on potato dextrose agar plates after 7 days at $30{ }^{\circ} \mathrm{C}$. The gold-coated dehydrated specimen was examined at different magnifications with Analytical Scanning Elec- 
tron Microscope Jeol JSM-6360 LA operating at $15 \mathrm{kV}$ at the Central Laboratory, City of Scientific Research and Technological Applications, Alexandria, Egypt. Then, molecular identification was applied.

Molecular identification. The selected isolate was subjected to molecular identification. The genomic DNA of B. cinerea SIB-1 was extracted utilizing the cetyltrimethylammonium bromide (CTAB) technique ${ }^{54}$ with some alterations. Mycelia of the selected isolate were scraped from the surface of a 4-day-old plate, homogenized in prewarmed CTAB extraction buffer, and incubated at $60^{\circ} \mathrm{C}$ for $30 \mathrm{~min}$. Cell debris was detached by centrifugation at 12,000 rpm for $20 \mathrm{~min}$, and the extracted DNA was purified from chromatin-associated protein by phenol/chloroform treatment. Then, DNA was precipitated by isopropanol, washed with $70 \%$ ethanol, and air-dried. The DNA pellet was redissolved in TE buffer and utilized as a template for PCR amplification.

Genomic DNA of $B$. cinerea SIB-1 was utilized as a template for PCR amplification of the ITS regions using primers ITS1 (5'-TCT GTA GGT GAA CCT GCG G-3') and ITS4 (5'-TCC TCC GCT TAT TGA TAT GC-3') described by White et $\mathrm{al} .{ }^{55}$. The specific primers were obtained from Clinilab (Clinilab Analysis Co., Egypt). The reaction mixture $(25 \mu \mathrm{l})$ contained $5 \mu \mathrm{l}$ of Taq red buffer, $20 \mathrm{ng}$ of template DNA, 10 pmol of each primer, 0.25 $\mathrm{U}$ of Taq polymerase (Bioline, UK). PCR cycling conditions were as follows: $95^{\circ} \mathrm{C}$ for $2 \mathrm{~min}, 30$ cycles of $95^{\circ} \mathrm{C}$ for $30 \mathrm{~s} ; 50{ }^{\circ} \mathrm{C}$ for $30 \mathrm{~min} ; 72^{\circ} \mathrm{C}$ for $1 \mathrm{~min}$; and 1 cycle of $72{ }^{\circ} \mathrm{C}$ for $5 \mathrm{~min}$. The PCR product was checked on a $1.5 \%$ agarose gel stained with $0.05 \mu \mathrm{g} / \mathrm{mL}$ ethidium bromide, and the target band was purified with a GF-1 PCR clean-up kit according to the manufacturer's guidelines.

The ITS sequence was computationally evaluated using the BLASTn program (http://www.ncbi.nlm.nih.gov/ BLAST). Sequences were aligned using Align Sequences Nucleotide BLAST. The obtained sequence was banked in GenBank to attain strictly related sequences; then, the accession number of the fungal strain was established. The evolutionary relationship was inferred, and the phylogenetic tree was generated using MEGA 10 software.

Antifungal activity of CNPs. Two tests were performed to investigate the in vitro antifungal activity of CNPs against the gray mold pathogen $B$. cinerea SIB-1. The first test was performed on agar plates containing PDA medium. The $\mathrm{pH}$ of the culture medium was adjusted prior to autoclaving using $1 \mathrm{M} \mathrm{NaOH}$ to be 6.5. So that, after sterilization and addition of CNPs or chitosan, the $\mathrm{pH}$ falls within the specified range (found to range from 5 to 5.5$)$.

Known amounts of CNPs were dissolved in acetic acid solution (1\%) with stirring overnight under aseptic conditions. The nanomaterial was added to PDA to ultimate concentrations of 0.5 and $1.0 \mathrm{mg} / \mathrm{mL}$. To prepare the fungal inoculum, $B$. cinerea SIB-1 mycelia were taken from a single colony and transferred to potato dextrose agar plates. The inoculated plates were incubated at $28^{\circ} \mathrm{C}$ for 7 days. A fungal disk $(5 \mathrm{~mm})$ was taken from the active growing margin and aseptically transferred to the center of the plates. The inoculated plates were checked daily until the control (zero CNPs) treatment touched the edge of the plate.

The in vitro antifungal activity of chitosan against $B$. cinerea SIB-1 was also investigated. On the other hand, the antifungal activity has been compared with the commercial Ridomil Gold fungicide on the mycelium growth of $B$. cinerea SIB-1 under the same conditions. Ridomil Gold is a leading fungicide recommended for controlling many important diseases on many crops. It is a mixture of both systemic (Mefenoxam) and contact (Mancozeb) fungicides to control certain diseases.

The second test was carried out to evaluate the protective effect of CNPs against gray mold disease. CNPs were prepared by centrifugation at $10,000 \mathrm{rpm}$ for $10 \mathrm{~min}$ and washed three times to remove any residue of acetic acid. Then, the washed CNPs were resuspended in sterile distilled water at concentrations ranging from 0 to $50 \mathrm{mg} /$ $\mathrm{mL}$. Healthy detached strawberry leaves were collected, and surface sterilized with $2 \%$ sodium hypochlorite for $3 \mathrm{~min}$ and $70 \%$ ethanol for $1 \mathrm{~min}$, rinsed with sterile water, and finally dried on filter paper. The collection of plant material has complied with relevant institutional, national, and international guidelines and legislation. The surface-sterilized leaves were then immersed in the nanoparticle solutions $(0,12.5,25$, and $50 \mathrm{mg} / \mathrm{mL})$ for $5 \mathrm{~min}$ and placed on moist paper towels in $9 \mathrm{~cm}$ Petri dishes with their adaxial surface up. Two mycelial plugs $(5 \mathrm{~mm})$ were acquired from the margin of a 5-day-old culture of $B$. cinerea SIB- 1 and adhered to the treated leaves, with five leaves for each treatment. The dishes were sealed with plastic film and incubated at $25^{\circ} \mathrm{C}$ in the dark for up to 5 days. The average percent of leaf area infected was determined for each concentration. Disease severity was evaluated in comparison to a disease severity scale of 0 (healthy leaf), 1 ( $<25 \%$ infection); $2=(25-50 \%$ infection) $3=(51-75 \%$ infection $)$, and $4(>75 \% \text { infection })^{56}$.

\section{Conclusions and challenges}

The current study introduced a novel biological-based approach for the green synthesis of chitosan nanoparticles using Pelargonium graveolens. Optimization of the bioprocess parameters was studied and maximized. All characterization tests on CNPs confirmed the suitability and efficacy of the plant as the bio-converter agent. The antifungal activity of the generated CNPs against a model phytopathogen with a wide host range (B. cinerea SIB-1) proved the ability of the CNPs to replace or minimize the extensive use of pesticides and to be applied in various technological and medical fields.

Although the data of the current study are promising, other several challenges are still recommended to be elucidated, such as the exact mode of action of the generated CNPs, the suitable inhibitory doses on a wider range of fungi, and the cytotoxicity of CNPs. However, the large-scale production of CNPs is another challenge before being approved as a commercial product.

Received: 1 October 2021; Accepted: 10 February 2022

Published online: 03 March 2022 


\section{References}

1. El-Naggar, N. E., Hussein, M. H. \& El-Sawah, A. A. Bio-fabrication of silver nanoparticles by phycocyanin, characterization, in vitro anticancer activity against breast cancer cell line and in vivo cytotxicity. Scientific Reports 7, 1-20 (2017).

2. Oh, J. W., Chun, S. C. \& Chandrasekaran, M. Preparation and in vitro characterization of chitosan nanoparticles and their broadspectrum antifungal action compared to antibacterial activities against phytopathogens of tomato. Agronomy 9, 21 (2019).

3. Yien, L., Zin, N. M., Sarwar, A. \& Katas, H. Antifungal activity of chitosan nanoparticles and correlation with their physical properties. Int. J. Biomater. 2012, 1-9 (2012).

4. Sakai, Y., Hayano, K., Yoshioka, H. \& Yoshioka, H. A novel method of dissolving chitosan in water for industrial application. Polym. J. 33, 640-642 (2001).

5. Huang, X. et al. Antifungal activity of chitosan against Phytophthora infestans, the pathogen of potato late blight. Int. J. Biol. Macromol. 166, 1365-1376 (2021).

6. Du, W. L., Niu, S. S., Xu, Y. L., Xu, Z. R. \& Fan, C. L. Antibacterial activity of chitosan tripolyphosphate nanoparticles loaded with various metal ions. Carbohydr. Polym. 75, 385-389 (2009).

7. Parveen, K., Banse, V. \& Ledwani, L., Green synthesis of nanoparticles: their advantages and disadvantages. in AIP conference proceedings. 1724, No. 1, p. 020048 (AIP Publishing LLC, 2016).

8. 8-Shankar, S. S., Ahmad, A., Pasricha, R., \& Sastry, M. Bioreduction of chloroaurate ions by geranium leaves and its endophytic fungus yields gold nanoparticles of different shapes. J. Mater. Chem. 13, 1822-1826 (2003).

9. El-Naggar, N. E., Hussein, M. H. \& El-Sawah, A. A. Phycobiliprotein-mediated synthesis of biogenic silver nanoparticles, characterization, in vitro and in vivo assessment of anticancer activities. Scientific Reports 8, 1-20 (2018).

10. Shankar, S. S., Rai, A., Ahmad, A. \& Sastry, M. Controlling the optical properties of lemongrass extract synthesized gold nanotriangles and potential application in infrared-absorbing optical coatings. Chem. Mater. 17, 566-572 (2005).

11. Abe, S. et al. Suppression of neutrophil recruitment in mice by geranium essential oil. Mediators Inflamm. 13, 21-24 (2004).

12. Ćavar, S. \& Maksimović, M. Antioxidant activity of essential oil and aqueous extract of Pelargonium graveolens L'Her. Food Control 23, 263-267 (2012).

13. Kochhar, S. L. Economic Botany (Cambridge University Press, 2016).

14. Boukhris, M. et al. Hypoglycemic and antioxidant effects of leaf essential oil of Pelargonium graveolens L.'Hér in alloxan induced diabetic rats. Lipids Health Dis. 11, 1-10 (2012).

15. Elad, Y., Williamson, B., Tudzynski, P. \& Delen, N. Botrytis spp and diseases they cause in agricultural systems: An introduction. In Botrytis: Biology, Pathology and Control 1-8 (Springer, 2007).

16. Fillinger, S. \& Elad, Y. (eds) Botrytis: The Fungus, the Pathogen and Its Management in Agricultural Systems 189-216 (Springer International Publishing, 2016).

17. Miclea, R. V., Suciu, L. A. \& Puia, C. E. In Vitro studies regarding the morphology of Botrytis Cinerea Pers. Isolated from geranium plants. Pro Environ. 5, 60-66 (2012).

18. El-Naggar, N. E. \& Abdelwahed, N. A. Application of statistical experimental design for optimization of silver nanoparticles biosynthesis by a nanofactory Streptomyces viridochromogenes. Journal of Microbiology 52, 53-63 (2014).

19. El-Naggar, N. E., Abdelwahed, N. A. \& Darwesh, O. M. Fabrication of biogenic antimicrobial silver nanoparticles by Streptomyces aegyptia NEAE 102 as eco-friendly nanofactory. Journal of Microbiology and Biotechnology 24, 453-464 (2014).

20. Kuppusamy, P., Yusoff, M. M., Maniam, G. P. \& Govindan, N. Biosynthesis of metallic nanoparticles using plant derivatives and their new avenues in pharmacological applications: An updated report. Saudi Pharm. J. 24, 473-484 (2016).

21. El-Naggar, N. E., Mohamedin, A., Hamza, S. S. \& Sherief, A.-D. Extracellular biofabrication, characterization, and antimicrobial efficacy of silver nanoparticles loaded on cotton fabrics using newly isolated Streptomyces sp. SSHH-1E. Journal of Nanomaterials (2016).

22. Mohamedin, A., El-Naggar, N. E., Shawqi Hamza, S. \& Sherief, A. Green synthesis, characterization and antimicrobial activities of silver nanoparticles by Streptomyces viridodiastaticus sshh-1 as a living nano-factory: Statistical optimization of process variables. Current Nanoscience 11, 640-654 (2015).

23. Das, R. K. et al. Biological synthesis of metallic nanoparticles: Plants, animals and microbial aspects. Nanotechnol. Environ. Eng. 2, 1-21 (2017).

24. Sathiyabama, M. \& Parthasarathy, R. Biological preparation of chitosan nanoparticles and its in vitro antifungal efficacy against some phytopathogenic fungi. Carbohydr. Polym. 151, 321-325 (2016).

25. El-Naggar, N. E. A., El-Khateeb, A. Y., Ghoniem, A. A., El-Hersh, M. S. \& Saber, W. I. Innovative low-cost biosorption process of $\mathrm{Cr}^{6+}$ by Pseudomonas alcaliphila NEWG-2. Sci. Rep. 10, 1-18 (2020).

26. Saber, W. I., El-Naggar, N. E., El-Hersh, M. S. \& El-Khateeb, A. Y. An innovative synergism between Aspergillus oryzae and Azotobacter chroococcum for bioconversion of cellulosic biomass into organic acids under restricted nutritional conditions using multi-response surface optimization. Biotechnology 14, 47-57 (2015).

27. Akita, T., Okumura, M., Tanaka, K., Kohyama, M. \& Haruta, M. Analytical TEM observation of Au nano-particles on cerium oxide. Catal. Today. 117, 62-68 (2006).

28. Fonseca, S. T., Nono, M. D. C. D. A. \& Cairo, C. A. A. Microstructure study of alumina ceramics from powder mixtures of nano and microsized particles. Mater. Sci. Forum 530, 661-664 (2006).

29. Vijayalakshmi, K., Devi, B. M., Sudha, P. N., Venkatesan, J. \& Anil, S. Synthesis, characterization and applications of nanochitosan/ sodium alginate/microcrystalline cellulose film. J. Nanomed. Nanotechnol. 7, 2 (2016).

30. Mohammed, M. A., Syeda, J., Wasan, K. M. \& Wasan, E. K. An overview of chitosan nanoparticles and its application in nonparenteral drug delivery. Pharmaceutics 9,53 (2017).

31. Corazzari, I. et al. Advanced physico-chemical characterization of chitosan by means of TGA coupled on-line with FTIR and GCMS: Thermal degradation and water adsorption capacity. Polym. Degrad. Stab. 112, 1-9 (2015).

32. Sivakami, M. S. et al. Preparation and characterization of nano chitosan for treatment wastewaters. Int. J. Biol. Macromol. 57, 204-212 (2013).

33. Ghadi, A., Mahjoub, S., Tabandeh, F. \& Talebnia, F. Synthesis and optimization of chitosan nanoparticles: Potential applications in nanomedicine and biomedical engineering. Caspian J. Intern. Med. 5, 156 (2014).

34. Zook, J. M., MacCuspie, R. I., Locascio, L. E., Halter, M. D. \& Elliott, J. T. Stable nanoparticle aggregates/agglomerates of different sizes and the effect of their size on hemolytic cytotoxicity. Nanotoxicology 5, 517-530 (2011).

35. Halamoda-Kenzaoui, B. et al. The agglomeration state of nanoparticles can influence the mechanism of their cellular internalisation. J. Nanobiotechnol. 15, 1-15 (2017).

36. Khan, I., Saeed, K. \& Khan, I. Nanoparticles: Properties, applications and toxicities. Arab. J. Chem. 12, 908-931 (2019).

37. Hanaor, D., Michelazzi, M., Leonelli, C. \& Sorrell, C. C. The effects of carboxylic acids on the aqueous dispersion and electrophoretic deposition of $\mathrm{ZrO}_{2}$. J. Eur. Ceram. Soc. 32, 235-244 (2012).

38. Kumar, A. \& Dixit, C. K. Methods for characterization of nanoparticles. In Advances in Nanomedicine for the Delivery of Therapeutic Nucleic Acids 43-58 (Woodhead Publishing, 2017).

39. Bozkir, A. \& Saka, O. M. Chitosan nanoparticles for plasmid DNA delivery: Effect of chitosan molecular structure on formulation and release characteristics. Drug Deliv. 11, 107-112 (2004).

40. Hassen, A. B. et al. Absolute line intensities for ethylene from 1800 to $2350 \mathrm{~cm}^{-1}$. J. Mol. Spectrosc. 282, 30-33 (2012) 
41. Silva, S. M. et al. Application of infrared spectroscopy to analysis of chitosan/clay nanocomposites. Infrared Spectrosc. 25, 43-62 (2012).

42. Jaworska, M., Kula, K., Chassary, P. \& Guibal, E. Influence of chitosan characteristics on polymer properties: II. Platinum sorption properties. Polym. Int. 52, 206-212 (2003).

43. Mahmoud, M. E., Abou Kana, M. T. \& Hendy, A. A. Synthesis and implementation of nano-chitosan and its acetophenone derivative for enhanced removal of metals. Int. J. Biol. Macromol. 81, 672-680 (2015).

44. Saber, W. I. A., Abd El-Hai, K. M. \& Ghoneem, K. M. Synergistic effect of Trichoderma and Rhizobium on both biocontrol of chocolate spot disease and induction of nodulation, physiological activities and productivity of Vicia faba. J. Microbiol. 4, 286-300 (2009).

45. Al-Askar, A. A., Saber, W. I., Ghoneem, K. M., Hafez, E. E. \& Ibrahim, A. A. Crude Citric acid of Trichoderma asperellum: Tomato growth promotor and suppressor of Fusarium oxysporum F. sp. lycopersici. Plants 10, 222 (2021).

46. Liu, X. F., Guan, Y. L., Yang, D. Z., Li, Z. \& Yao, K. D. Antibacterial action of chitosan and carboxymethylated chitosan. J. Appl. Polym. Sci. 79, 1324-1335 (2001).

47. Al-Hetar, M. Y., Zainal Abidin, M. A., Sariah, M. \& Wong, M. Y. Antifungal activity of chitosan against Fusarium oxysporum f. sp. cubense. J. Appl. Polym. Sci. 120(4), 2434-2439 (2011).

48. Birch, P. R. et al. Oomycete RXLR effectors: Delivery, functional redundancy and durable disease resistance. Curr. Opin. Plant Biol. 11, 373-379 (2008).

49. Greyerbiehl, J. A. Induction of Resistance in Potato Tuber Tissue to Control Fusarium sambucinum (Michigan State University, 2002).

50. Van, S. N., Minh, H. D. \& Anh, D. N. Study on chitosan nanoparticles on biophysical characteristics and growth of Robusta coffee in green house. Biocatal. Agric. Biotechnol. 2, 289-294 (2013).

51. Asgari-Targhi, G., Iranbakhsh, A. \& Ardebili, Z. O. Potential benefits and phytotoxicity of bulk and nano-chitosan on the growth, morphogenesis, physiology, and micropropagation of Capsicum annuum. Plant Physiol. Biochem. 127, 393-402 (2018).

52. Gu, C., Sun, B., Wu, W., Wang, F. \& Zhu, M. Synthesis characterization of copper-loaded carboxymethyl-chitosan nanoparticles with effective antibacterial activity. Macromol. Symp. 254, 160-166 (2007).

53. Khan, I., Abdalla, A. \& Qurashi, A. Synthesis of hierarchical $\mathrm{WO}_{3}$ and $\mathrm{Bi}_{2} \mathrm{O}_{3} / \mathrm{WO}_{3}$ nanocomposite for solar-driven water splitting applications. Int. J. Hydrog. Energy 42, 3431-3439 (2017).

54. Doyle, J. J. \& Doyle, J. L. A rapid DNA isolation procedure for small quantities of fresh leaf tissue. Phytochem. Bull. 19, 11-15 (1987).

55. White, T. J., Bruns, T., Lee, S. \& Taylor, J. Amplification and direct sequencing of fungal ribosomal RNA genes for phylogenetics. In PCR Protocols: A Guide to Methods and Applications (eds Innis, A. et al.) 315-322 (Academic Press, 1990).

56. Segarra, G., Casanova, E., Borrero, C., Avilés, M. \& Trillas, I. The suppressive effects of composts used as growth media against Botrytis cinerea in cucumber plants. Eur. J. Plant Pathol. 117, 393-402 (2007).

\title{
Acknowledgements
}

The authors gratefully acknowledge the City of Scientific Research and Technological Applications (SRTA-City), Alexandria, 21934, Egypt, for providing financial support for laboratory measurements and analyzes of this paper within the framework of SRTA-City Central Laboratories Services.

\section{Author contributions}

N.E.E. proposed the research topic, designed the research plan, provided necessary tools for experiments and experimental instructions, collected the data, performed the statistical analysis, and contributed to the review and revision of the manuscript. W.I.S. interpretation of the results and writing and revision of the manuscript. A.M.Z. carried out some of the experiments. S.I.B. carried out all the biological control experiments, identification of Botrytis cinerea SIB-1 and contributed to the writing and revision of the manuscript. All authors read and approved the final manuscript.

\section{Funding}

Open access funding provided by The Science, Technology \& Innovation Funding Authority (STDF) in cooperation with The Egyptian Knowledge Bank (EKB).

\section{Competing interests}

The authors declare no competing interests.

\section{Additional information}

Supplementary Information The online version contains supplementary material available at https://doi.org/ 10.1038/s41598-022-07073-y.

Correspondence and requests for materials should be addressed to N.E.-A.E.-N.

Reprints and permissions information is available at www.nature.com/reprints.

Publisher's note Springer Nature remains neutral with regard to jurisdictional claims in published maps and institutional affiliations.

\begin{abstract}
Open Access This article is licensed under a Creative Commons Attribution 4.0 International License, which permits use, sharing, adaptation, distribution and reproduction in any medium or
format, as long as you give appropriate credit to the original author(s) and the source, provide a link to the Creative Commons licence, and indicate if changes were made. The images or other third party material in this article are included in the article's Creative Commons licence, unless indicated otherwise in a credit line to the material. If material is not included in the article's Creative Commons licence and your intended use is not permitted by statutory regulation or exceeds the permitted use, you will need to obtain permission directly from the copyright holder. To view a copy of this licence, visit http://creativecommons.org/licenses/by/4.0/.
\end{abstract}

(C) The Author(s) 2022 